\title{
Bifurcation of bounded solutions of impulsive differential equations
}

\author{
Kevin E.M. Church \\ Department of Applied Mathematics, University of Waterloo \\ Waterloo, Ontario, N2L 3G1, Canada, \\ k5church@uwaterloo.ca \\ Xinzhi Liu \\ Department of Applied Mathematics, University of Waterloo \\ Waterloo, Ontario, N2L 3G1, Canada, \\ xzliu@uwaterloo.ca
}

\begin{abstract}
In this article, we examine nonautonomous bifurcation patterns in nonlinear systems of impulsive differential equations. The approach is based on Lyapunov-Schmidt reduction applied to the linearization of a particular nonlinear integral operator whose zeroes coincide with bounded solutions of the impulsive differential equation in question. This leads to sufficient conditions for the presence of fold, transcritical and pitchfork bifurcations. Additionally, we provide a computable necessary condition for bifurcation in nonlinear scalar impulsive differential equations. Several examples are provided illustrating the results.

Keywords: Impulsive differential equations, bifurcation, bounded solution, hyperbolicity, exponential dichotomy
\end{abstract}

\section{Introduction}

Impulsive differential equations have become increasingly more relevant in applications in recent years. Optimal control, mathematical biology and chemical kinetics applications abound [Church \& Smith?, 2016; Galbusera \& Pasquali, 2015; Li, Feng \& Wang, 2009; Zhao \& Chen, 2009; Zhao, Yang \& Chen, 2009], this subfield of discontinuous dynamics has grown wide and has been the subject of a great deal of theoretical research. However, research into bifurcation theory of impulsive systems has been modest. Although there are numerous examples [Church \& Smith?, 2016; Georgescu, Zhang \& Chen, 2008; Zhao, Yang \& Chen, 2009] of the techniques of autonomous bifurcation theory of discrete-time systems being applied to periodic systems of impulsive differential equations, there are few elementary results that are suitable for general aperiodic systems. Positive results in more general directions include [Akhmet \& Kashkynbayev, 2013, 2016], in which the authors considered Bernoulli-type equations and studied bifurcation of solutions based on notions of pullback stability and attraction, much in the same vein as Langa et. al [Langa, Robinson \& Suarez, 2002, 2006]. In [Akhmet \& Kashkynbayev, 2013], the authors obtain linearized past-, forward-, and all-time attractivity conditions for systems of impulsive differential equations and obtain some elementary bifurcation results in the sense of nonautonomous bifurcations as pioneered by Rasmussen [Rasmussen, 2007] and others. Still another direction exploits Rabbinowitz global bifurcation theorems to describe the global structure of solutions of impulsive boundary value problems [Liu, 2015; Niu \& Yan, 2016; Liu \& O'Reagan, 2011], obtaining sufficient conditions for multiplicity of solutions and bifurcation points and intervals. 
In the present article, we will focus our attention on the methods of static bifurcation theory. Recently [Pötzsche, 2010], by means of Lyapunov-Schmidt reduction, abstract bifurcation conditions for differential equations in Banach spaces have been determined under the assumption that, at the critical parameter, the variational equation about a given reference solution of interest lacks an exponential dichotomy, but posesses exponential dichotomies on half-lines. Our objective will be to extend this result to systems of impulsive differential equations, although we choose to remain in the more concrete finite-dimensional setting of Euclidean space (though one could certainly obtain analogous results in a Banach space setting).

The paper is organized as follows. In Section 2, we introduce some of the basic definitions and notation that will be used throughout. Section 3 will be devoted to the introduction of a nonlinear integral operator and its properties: namely, smoothness properties and the connection between its zeroes and solutions of a given impulsive differential equation. Section 4 pertains to fundamental properties of the linearization of the operator defined in Section 3. Namely, bifurcation points are characterized and Fredholm properties are determined. Section 5 provides several sufficient conditions for classical bifurcation patterns. Section 6 contains an analytically precise necessary condition for bifurcation of bounded solutions in nonlinear scalar systems, with some examples. The penultimate Section 7 contains examples of bifurcations in planar systems that take advantage of the results from Section 5. We end (Section 8) with a conclusion.

\section{Background}

We begin by defining symbols and function spaces that will be seen throughout. The nonnegative real numbers will be denoted $\mathbb{R}_{+}$, and the nonpositive real numbers by $\mathbb{R}_{-} \cdot|\cdot|$ is the Euclidean norm on $\mathbb{R}^{n}$, $\|\cdot\|$ is the induced operator norm, and $\|f\|_{0}=\sup _{t \in \mathbb{R}}|f(t)|$ for a function $f: \mathbb{R} \rightarrow \mathbb{R}^{n}$. The symbols $\mathcal{R} L$ and $\mathcal{N} L$ denote the range and kernel of a linear operator $L$. $\Omega$ will denote a convex open subset of $\mathbb{R}^{n}, \Lambda$ an open subset of $\mathbb{R}^{m}$. The interior of a set $X$ is denoted $X^{\circ}$, and $\# X$ denotes the cardinality of $X$. For a topological vector space $V$, the symbol $V^{*}$ denotes its continuous dual.

Let $\tau_{k}$ be a real, increasing sequence unbounded on every compact set. Introduce the function space

$$
P C(\Omega)=\left\{\begin{array}{l}
f: \mathcal{I} \rightarrow \Omega \mid f \text { is continuous except at times } \tau_{k}, \text { where it is continuous } \\
\text { from the left and has limits on the right }
\end{array}\right\}
$$

The notation $\dot{f}$ indicates the left-derivative of $f$. Next, introduce the spaces

$$
\begin{aligned}
B P C(\Omega) & =\left\{f \in P C(\mathbb{R}, \Omega):\|f\|_{0}<\infty\right\}, \\
B P C^{1}(\Omega) & =\left\{f \in B P C(\Omega): \dot{f} \in B P C\left(\mathbb{R}^{n}\right)\right\} .
\end{aligned}
$$

Then,

$$
\|f\|_{1}=\max \left\{\|f\|_{0},\|\dot{f}\|_{0}\right\}
$$

is a norm on $B P C^{1}(\Omega)$. Also, we will denote $B P C^{1}=B P C^{1}\left(\mathbb{R}^{n}\right)$. The space $B P C\left(\mathbb{R}^{n}\right)$ with the norm $\|\cdot\|_{0}$ is complete [Akhmet, 2010], and it is a straightforward exercise to prove that the same holds for $B P C^{1}$ with the norm $\|\cdot\|_{1}$.

Our object of interest will be an impulsive differential equation

$$
\begin{aligned}
\dot{x} & =f(t, x, \lambda), \quad t \neq \tau_{k} \\
\Delta x & =J_{k}(x, \lambda), \quad t=\tau_{k},
\end{aligned}
$$

where $f: \mathbb{R} \times \Omega \times \Lambda \rightarrow \mathbb{R}^{n}, J_{k}: \Omega \times \Lambda \rightarrow \mathbb{R}^{n}$, and $\tau_{k} \in \mathbb{R}$ is a strictly increasing real sequence of impulse times that is unbounded on $\mathbb{R}_{+}$and $\mathbb{R}_{-}$.

We will also be interested in homogeneous linear impulsive differential equations

$$
\begin{aligned}
\dot{x} & =A(t) x, & & t \neq \tau_{k} \\
\Delta x & =B_{k} x, & & t=\tau_{k},
\end{aligned}
$$

for $A(t), B_{k} \in \mathbb{R}^{n \times n}$. The transition operator is the unique matrix-valued function $X(t, s)$, defined for $t \geq s$, such that $x=X(t, s) x_{s}$ is the unique solution of the initial-value problem $x(s)=x_{s}$ for $(1)-(2)$ defined on $[s, \infty]$. We will say that $X(t, s)$ is reversible if $X(t, s)^{-1}$ exists for all $t \geq s$. In this case, we 
write $X(t, s)^{-1}=X(s, t)$. Note that, generally speaking, when $A(t)$ is regular enough, $X(t, s)$ is reversible if and only if $\operatorname{det}\left(I+B_{k}\right)_{k \in \mathbb{Z}} \neq 0$.

Definition 2.1. We say that the homogeneous impulsive differential equation (1)-(2), or $X(t, s)$, has an exponential dichotomy on $\mathcal{I} \subseteq \mathbb{R}$ if there exist a projection $P(t)$, satisfying $X(t, s) P(s)=P(t) X(t, s)$ for $t \geq s$ in $\mathcal{I}$, and there exist constants $K \geq 1$ and $\alpha>0$ such that

$$
\begin{aligned}
|X(t, s) P(s)| & \leq K e^{-\alpha(t-s)}, & & s \leq t \in \mathcal{I} \\
|X(s, t)[I-P(t)]| & \leq K e^{-\alpha(t-s)}, & & s \leq t \in \mathcal{I} .
\end{aligned}
$$

It is well-known [Bainov \& Simeonov, 1993; Kiskinov et. al, 2015] that if $X(t, s)$ posesses an exponential dichotomy, then so does the matrix $Y(t, s)=X^{*}(s, t)$, which coincides with transition operator for the adjoint system

$$
\begin{aligned}
\dot{y} & =-A^{*}(t) y, & & t \neq \tau_{k} \\
\Delta y & =-B_{k}^{*}\left(I=B_{k}^{*}\right)^{-1} y, & & t=\tau_{k} .
\end{aligned}
$$

We summarize this in the following proposition whose proof we omit.

Proposition 1. Let $X(t, s)$ posess an exponential dichotomy on $\mathcal{I}$ with exponent $\alpha$ and projector $P$. Then $Y(t, s)=X^{*}(s, t)$ posesses an exponential dichotomy on $\mathcal{I}$ with exponent $\alpha$ and projector $I-P^{*}$. Specifically, we have the following:

$$
\begin{aligned}
\left|Y(t, s)\left[I-P^{*}(s)\right]\right| & \leq K e^{-\alpha(s-t)}, & & s \leq t \in \mathcal{I}, \\
\left|Y(s, t) P^{*}(t)\right| & \leq K e^{-\alpha(t-s)}, & & s \leq t \in \mathcal{I} .
\end{aligned}
$$

Remark 2.1. The definition of exponential dichotomy assumes $X(t, s)$ is reversible. The definition can be modified so that an exponential dichotomy can be defined in the non-reversible case by imposing that the restriction of $X(t, s)$ to $\mathcal{R} P(s)$ is an isomorphism onto $\mathcal{R} P(t)$. Dichotomy properties are still carried over to the adjoint matrix (generally, dual operator). See [Pötzsche, 2010] or [Ruan \& Zhang, 2005] for the idea.

\section{The solution operator}

We begin by defining a formal integral operator whose zeroes will coincide with solutions of the impulsive differential equation $\left(E_{\lambda}\right)$. We begin with a definition.

Definition 3.1. Given an impulsive differential equation $\left(E_{\lambda}\right)$, the $(\mathbf{A}, \mathbf{B})$-augmented equation, for a continuous $\mathbf{A}(t) \in \mathbb{R}^{n \times n}$ and $\mathbf{B}=\mathbf{B}_{k \in \mathbb{Z}}$, is the formal semilinear impulsive differential equation

$$
\begin{aligned}
\dot{x} & =\mathbf{A}(t) x+\mathbf{f}(t, x, \lambda), & & t \neq \tau_{k} \\
\Delta x & =\mathbf{B}_{k} x+\mathbf{J}_{k}(x, \lambda), & & t=\tau_{k},
\end{aligned}
$$

with $\mathbf{f}=f-\mathbf{A} x$ and $\mathbf{J}=J-\mathbf{B} x$. The associated homogeneous equation is the impulsive differential equation

$$
\begin{aligned}
\dot{x} & =\mathbf{A}(t) x, \quad t \neq \tau_{k} \\
\Delta x & =\mathbf{B}_{k} x, \quad t=\tau_{k},
\end{aligned}
$$

and its transition operator is denoted $\mathbf{X}(t, s)$.

In the following sections, we will work with a fixed a pair $(\mathbf{A}, \mathbf{B})$, the augmented equation, and the associated transition operator $\mathbf{X}(t, s)$ for the homogeneous equation. Define a formal nonlinear operator $H: B P C^{1}(\mathbb{R}, \Omega) \times \Lambda \rightarrow B P C^{1}$ by the following formula:

$$
H:(\phi, \lambda)[t]=\phi(t)-\left[\int_{-\infty}^{t} \mathbf{X}(t, s) \mathbf{f}(s, \phi(s), \lambda) d s+\sum_{\tau_{k}<t} \mathbf{X}\left(t, \tau_{k}^{+}\right) \mathbf{J}_{k}\left(\phi\left(\tau_{k}\right), \lambda\right)\right] .
$$

The following assumptions will be needed throughout. 
H0. $\mathbf{A}$ is continuous, $\sup _{t \in \mathbb{R}}\|\mathbf{A}(t)\|=\|\mathbf{A}\|<\infty$, and $\mathbf{X}(t, s)$ has an exponential dichotomy on $\mathbb{R}$ with projection $P=I$. That is, there exists $K \geq 1$ and $\alpha>0$ such that $|\mathbf{X}(t, s)| \leq K e^{-\alpha(t-s)}$ for $t \geq s$.

H1. Let $m \in \mathbb{N}$, and suppose $f: \mathbb{R} \times \Omega \times \Lambda \rightarrow X$ and $J_{k}: \Omega \times \Lambda \rightarrow X$ are continuous and the partial derivatives $D_{(2,3)}^{j} f$ and $D_{(1,2)}^{j} J_{k}$ are continuous, for $0 \leq j \leq m$ and $k \in \mathbb{Z}$, and that they satisfy the following continuity and boundedness conditions for all bounded $C \subseteq \Omega$ :

$$
\sup _{t \in \mathbb{R}} \sup _{u \in C}\left|D_{(2,3)}^{j} f(t, u, \lambda)\right|<\infty, \quad \sup _{k \in \mathbb{Z} u \in C}\left|D_{(1,2)}^{j} J_{k}(u, \lambda)\right|<\infty, \quad \lambda \in \Lambda,
$$

and for all $\lambda^{*} \in \Lambda$ and $\epsilon>0$, there exists $\delta>0$ such that $\|u-\bar{u}\|_{0}<\delta$ implies

$$
\left\|D_{(2,3)}^{j} f(t, u, \lambda)-D_{(2,3)}^{j}(t, \bar{u}, \lambda)\right\|_{0}<\epsilon \text {, and }\left\|D_{(1,2)}^{j} J_{k}(u, \lambda)-D_{(1,2)}^{j} J_{k}(\bar{u}, \lambda)\right\|_{0}<\epsilon,
$$

for all $u, \bar{u} \in \Omega$ and $\lambda \in B_{\delta}\left(\lambda^{*}\right)$.

H2. There exists $b>0$ such that $\tau_{k+1}-\tau_{k} \geq b$ for all $k \in \mathbb{Z}$.

Our first results concern the well-definition of the operator $H$ and its smoothness. To begin, define the formal derivatives, for integers $j \in\{0, \ldots, m\}$,

$$
G^{j}(\phi, \lambda)[X]=\int_{-\infty}^{t} \Phi(t, s) D_{(2,3)}^{j} f(s, \phi(s), \lambda) X(s) d s+\sum_{\tau_{k}<t} \Phi\left(t, \tau_{k}^{+}\right) D_{(1,2)}^{j} J_{k}\left(\phi\left(\tau_{k}\right), \lambda\right) X\left[\tau_{k}\right]
$$

and the formal partial derivatives, for $v=\left(v_{1}, v_{2}\right) \in \mathbb{N}_{0}^{2}$ with $v_{1}+v_{2} \leq m$,

$$
G^{v}(\phi, \lambda)[X]=\int_{-\infty}^{t} \Phi(t, s) D_{2}^{v_{1}} D_{3}^{v_{2}} f(s, \phi(s), \lambda) X[s] d s+\sum_{\tau_{k}<t} \Phi\left(t, \tau_{k}^{+}\right) D_{1}^{v_{1}} D_{2}^{v_{2}} J_{k}\left(\phi\left(\tau_{k}\right), \lambda\right) X\left[\tau_{k}\right],
$$

where $X$ represents an element of an appropositionriate product space of the form $L_{j}\left(B P C^{1}(\Omega) \times \Lambda, B P C^{1}\right)$ or $L_{v_{1}}\left(B P C^{1}(\Omega), L_{v_{2}}\left(\Lambda, B P C^{1}\right)\right)$, and for an operator $L$ acting on one of these spaces, we denote $L X(s)=$ $(L X)(s)$ for the post-operarational evaluation in $B P C^{1}$.

Lemma 1. Under conditions HO-H2, the operators

$$
\begin{aligned}
& G^{j}: B P C^{1}(\mathbb{R}, \Omega) \times \Lambda \rightarrow\left(L_{j}\left(B P C^{1} \times \Lambda, B P C^{1}\right)\right. \\
& G^{v}: B P C^{1}(\mathbb{R}, \Omega) \times \Lambda \rightarrow L_{v_{1}}\left(B P C^{1}, L_{v_{2}}(\Lambda, X)\right)
\end{aligned}
$$

are well-defined and continuous.

Proof. In the following, we will suppress the variable $X$ appearing in equations (7) and (8). Also, we prove only the result for $G^{j}$ and integers $j$. First, we prove that $G^{j}$ is well-defined. For each $t \in \mathbb{R}$, we have

$$
\begin{aligned}
\left|G^{j}(\phi, \lambda)[t]\right| \leq & \int_{-\infty}^{t}\left|\mathbf{X}(t, s) D_{(2,3)}^{j} \mathbf{f}(s, \phi(s), \lambda)\right| d s+\sum_{\tau_{k}<t}\left|\mathbf{X}\left(t, \tau_{k}^{+}\right) D_{(1,2)}^{j} \mathbf{J}_{k}\left(\phi\left(\tau_{k}\right), \lambda\right)\right| \\
& \leq \int_{-\infty}^{t} K e^{-\alpha(t-s)} c_{1} d s+\sum_{\tau_{k}<t} K e^{-\alpha\left(t-\tau_{k}\right)} c_{2} \\
& \leq \frac{K c_{1}}{\alpha}+\frac{K c_{2}}{1-e^{-\alpha b}}
\end{aligned}
$$

$c_{1}$ and $c_{2}$ are finite constants guaranteed by hypothesis $\mathrm{H} 1, K$ and $\alpha$ are as in $\mathrm{H} 0$, and $b$ is as defined in H2. and we have assume without loss of generality that $\tau_{k}$ is an unbounded sequence of impulses. It follows that the integrals and sums defining $G^{j}$ are absolutely integrable and summable, so $t \mapsto G^{j}(\phi, \lambda)[t]$ indeed exists. Moreover, $G^{j}(\phi, \lambda)$ is globally bounded. One can prove by similar estimations that it is continuous. 
Next, we prove that $G^{j}(\phi, \lambda) \in B P C^{1}$. Existence of the left derivative is fully justified by hypothesis $\mathrm{H} 1$ and the properties of the fundamental matrix $\mathbf{X}(t, s)$. In particular, we have

$$
\frac{d}{d t} G^{j}(\phi, \lambda)[t]=D_{(2,3)}^{j} \mathbf{f}(t, \phi(t), \lambda)+\mathbf{A}(t) G^{j}(\phi, \lambda)[t],
$$

and due to boundedness of $A(t)$ guaranteed by assumption (H4), we conclude that $G^{j}(\phi, \lambda) \in B P C^{1}$.

We now prove continuity of $(\phi, \lambda) \mapsto G^{j}(\phi, \lambda)$. Let $\epsilon$ and $\delta$ be as in H1. We find

$$
\begin{aligned}
\left\|G^{j}(\phi, \lambda)-G^{j}(\psi, \mu)\right\|_{0} \leq & \sup _{t \in \mathbb{R}} \int_{-\infty}^{t}\left|\mathbf{X}(t, s)\left[D^{j} \mathbf{f}(s, \phi(s), \lambda)-D^{j} \mathbf{f}(s, \psi(s), \mu)\right]\right| d s \\
& +\sup _{t \in \mathbb{R}} \sum_{\tau_{k}<t}\left|\mathbf{X}\left(t, \tau_{k}^{+}\right)\left[D^{j} \mathbf{J}_{k}\left(\phi\left(\tau_{k}\right), \lambda\right)-D^{j} \mathbf{J}_{k}\left(\psi\left(\tau_{k}\right), \mu\right)\right]\right| \\
& \leq \sup _{t \in \mathbb{R}}\left(\int_{-\infty}^{t} K e^{-\alpha(t-s)} \epsilon d s+\sum_{\tau_{k}<t} K e^{-\alpha\left(t-\tau_{k}\right)} \epsilon\right) \\
& \leq \epsilon K\left(\frac{1}{\alpha}+\frac{1}{1-e^{-\alpha b}}\right):=\epsilon C
\end{aligned}
$$

Also,

$$
\begin{aligned}
\left\|\frac{d}{d t} G^{j}(\phi, \lambda)-\frac{d}{d t} G^{j}(\psi, \mu)\right\| \leq & \sup _{t \in \mathbb{R}}\left(\left|D_{(2,3)}^{j} \mathbf{f}(t, \phi(t), \lambda)-D_{(2,3)}^{j} \mathbf{f}(t, \psi(t), \mu)\right|\right. \\
& \left.+|\mathbf{A}(t)| \cdot\left|G^{j}(\phi, \lambda)[t]-G^{j}(\psi, \mu)[t]\right|\right) \\
& \leq \epsilon+\|\mathbf{A}\| \cdot\left\|G^{j}(\phi, \lambda)-G^{j}(\psi, \lambda)\right\|_{0} \\
& \leq \epsilon(1+\|\mathbf{A}\| C)
\end{aligned}
$$

Therefore, $G^{j}$ is continuous.

Corollary 3.1. Let hypotheses HO-H2 be satisfied. The operator H defined in equation (6) is well-defined and continuous.

Proposition 2. Let hypotheses H0-H2 be satisfied. The operator $G: B P C(\Omega) \times \Lambda \rightarrow B P C$ is well-defined and $m$-times continuously differentiable on $B P C(\Omega)^{\circ} \times \Lambda$, with partial derivatives

$$
D^{v} G(\phi, \lambda)=G^{v}(\phi, \lambda)
$$

Proof. We know that each of the operators $G^{v}$ is well-defined and continuous for $v=\left(v_{1}, v_{2}\right), v_{1}+v_{2} \leq m$, due to Lemma 1. We prove only that $D^{j} G=G^{j}$ for integers $j \in\{0, \ldots, m-1\}$, since the result pertaining to partial derivatives then follows by definition of Frechet differentiability.

Let $\phi^{*} \in B P C^{1}(\Omega)^{\circ}$, let $\lambda, \lambda^{*} \in \Lambda$, and let $\phi \in B P C^{1}$ be small enough so that $\phi^{*}+h \phi \in B P C^{1}(\Omega)^{\circ}$ for all $h \in[0,1]$. For each $j \in\{0, \ldots, m-1\}$, define the remainder

$$
r_{j}(\phi, \lambda)=\sup _{h, s \in[0,1]}\left\|F^{j+1}\left(\phi^{*}+h \phi, \lambda^{*}+h \lambda\right)-F^{j+1}\left(\phi^{*}, \lambda^{*}\right)\right\|_{0}+\left\|J^{j+1}\left(\phi^{*}+s \phi, \lambda^{*}+s \lambda\right)-J^{j+1}\left(\phi^{*}, \lambda^{*}\right)\right\|_{0}^{\mathbb{Z}},
$$

where $F^{j}(\phi, \lambda)[t]=D_{(2,3)}^{j} \mathbf{f}(t, \phi, \lambda)$ and $J^{j}(\phi, \lambda)[k]=D_{(1,2)}^{j} \mathbf{J}_{k}\left(\phi\left(\tau_{k}\right), \lambda\right)$. Continuity of $F^{j+1}$ and $J^{j+1}$ are guaranteed by hypothesis H1, so we have that $r_{j}(\phi, \lambda) \rightarrow 0$ as $(\phi, \lambda) \rightarrow 0$. By the mean-value theorem [Lang, 1993]

$$
\begin{aligned}
& \left|F^{j}\left(\phi^{*}+\phi, \lambda^{*}+\lambda\right)[t]-F^{j}\left(\phi^{*}, \lambda^{*}\right)[t]-F^{j+1}\left(\phi^{*}, \lambda^{*}\right)[t][\phi \lambda]^{T}\right| \\
& \leq \int_{0}^{1}\left|F^{j+1}\left(\phi^{*}+h \phi, \lambda^{*}+h \lambda\right)[t]-F^{j+1}\left(\phi^{*}, \lambda^{*}\right)[t]\right| d h\left|[\phi \lambda]^{T}\right| \\
& \leq r_{j}(\phi, \lambda)\left|[\phi \lambda]^{T}\right|,
\end{aligned}
$$


and similarly,

$$
\left|D^{j} J_{k}\left(\phi^{*}+\phi, \lambda^{*}+\lambda\right)-D^{j} J_{k}\left(\phi^{*}, \lambda^{*}\right)-D^{j+1} J_{k}\left(\phi^{*}, \lambda^{*}\right)\left[\phi\left(\tau_{k}\right) \lambda\right]^{T}\right| \leq r_{j}(\phi, \lambda)\left|[\phi \lambda]^{T}\right| .
$$

By passing to suprema in $t$ and $k$,

$$
\begin{aligned}
& \left\|G^{j}\left(\phi^{*}+\phi, \lambda^{*}+\lambda\right)-G^{j}\left(\phi^{*}, \lambda^{*}\right)-G^{j+1}\left(\phi^{*}, \lambda^{*}\right)[\phi \lambda]^{T}\right\|_{0} \\
& \leq \sup _{t \in \mathbb{R}}\left(\int_{-\infty}^{t} K e^{-r(t-s)} d s+\sum_{\tau_{k}<t} K e^{-r\left(t-\tau_{k}\right)}\right) r_{j}(\phi, \lambda) \max \{\|\phi\|,\|\lambda\|\} \\
& \leq K\left(\frac{1}{\alpha}+\frac{1}{1-e^{-\alpha b}}\right) r_{j}(\phi, \lambda)\|(\phi, \lambda)\|_{1} .
\end{aligned}
$$

On the other hand, we have

$$
\begin{aligned}
& \left|D^{j} \dot{f}\left(t, \phi^{*}+\phi, \lambda^{*}+\lambda\right)-D^{j} \dot{f}\left(t, \phi^{*}, \lambda^{*}\right)-D^{j+1} \dot{f}\left(t, \phi^{*}, \lambda^{*}\right)[\phi \lambda]^{T}\right| \\
& \leq \int_{0}^{1}\left|D^{j+1} \dot{f}\left(t, \phi^{*}+h \phi, \lambda^{*}+h \lambda\right)-D^{j+1} \dot{f}\left(t, \phi^{*}, \lambda^{*}\right)\right| d h\left|[\phi \lambda]^{T}\right| \\
& \leq r_{j}(\phi, \lambda)\left|[\phi \lambda]^{T}\right| .
\end{aligned}
$$

Using equation (9), we can obtain

$$
\begin{aligned}
& \frac{d}{d t}\left(G^{j}\left(\phi^{*}+\phi, \lambda^{*}+\lambda\right)-G^{j}\left(\phi^{*}, \lambda^{*}\right)-G^{j+1}\left(\phi^{*}, \lambda^{*}\right)[\phi \lambda]^{T}\right) \\
& =F^{j}\left(\phi^{*}+\phi, \lambda^{*}+\lambda\right)[t]-F^{j}\left(\phi^{*}, \lambda^{*}\right)[t]-F^{j+1}\left(\phi^{*}, \lambda^{*}\right)[t][\phi \lambda]^{T} \\
& \quad+\mathbf{A}(t)\left(G^{j}\left(\phi^{*}+\phi, \lambda^{*}+\lambda\right)-G^{j}\left(\phi^{*}, \lambda^{*}\right)-G^{j+1}\left(\phi^{*}, \lambda^{*}\right)[\phi \lambda]^{T}\right) .
\end{aligned}
$$

Consequently,

$$
\left\|\partial_{t} G^{j}\left(\phi^{*}+\phi, \lambda^{*}+\lambda\right)-\partial_{t} G^{j}\left(\phi^{*}, \lambda^{*}\right)-\partial_{t} G^{j+1}\left(\phi^{*}, \lambda^{*}\right)[\phi \lambda]^{T}\right\|_{0} \leq C r_{j}(\phi, \lambda)\|(\phi, \lambda)\|_{1},
$$

where $C=\left(1+\|\mathbf{A}\|_{0} K\left(\alpha^{-1}+\left(1-e^{-\alpha b}\right)^{-1}\right)\right)$. We conclude that $G^{j}$ is differentiable and $D_{(2,3)} G^{j}=G^{j+1}$, as required.

Finally, we arrive at our smoothness result for the operator $H$, which is a direct corollary of the above lemma.

Corollary 3.2. Under hypotheses H0-H2, the operator $H: B P C^{1}(\Omega) \times \Lambda \rightarrow B P C^{1}$ defined in (6) is $m$-times continuously differentiable on $B P C^{1}(\Omega)^{\circ} \times \Lambda$.

We have a result concerning the solutions of the impulsive differential equation $\left(E_{\lambda}\right)$ and zeroes of the operator $H$.

Theorem 1. Let $\lambda \in \Lambda$. Suppose hypotheses HO-H2 hold. $\phi: \mathbb{R} \rightarrow \Omega$ is a bounded, complete solution of $\left(E_{\lambda}\right)$ if and only if

$$
H(\phi, \lambda)=0
$$

for the operator $H: B P C^{1}(\Omega) \times \Lambda \rightarrow B P C^{1}$ defined in (6).

Proof. Suppose $\phi \in B P C^{1}(\Omega)$ is a complete solution of solves $\left(E_{\lambda}\right)$. Then, $\phi$ is a complete solution of $\left(\mathbf{E}_{\lambda}\right)$, and so by the variation of constants formula, the following is valid for $t \geq y$ :

$$
\phi(t)=\mathbf{X}(t, y) \phi(y)+\int_{y}^{t} \mathbf{X}(t, s) \mathbf{f}(s, \phi(s), \lambda) d s+\sum_{y \leq \tau_{k}<t} \mathbf{X}\left(t, \tau_{k}^{+}\right) \mathbf{J}_{k}\left(\phi\left(\tau_{k}\right), \lambda\right) .
$$


The left-hand side is independent of $y$, and $\phi(y)$ is bounded as $y \rightarrow-\infty$. Therefore, taking the aforementioned limit, assumption $\mathrm{H} 0$ and Corollary 3.1 imply that $\phi(t)=H(\phi, \lambda)[t]$ for all $t \in \mathbb{R}$, so that equation (10) is satisfied.

Conversely, if $\phi$ satisfies equation (10), taking time derivatives on both sides of the equation shows that $\phi$ satisfies the differential equation of $\left(\mathbf{E}_{\lambda}\right)$ at times $t \neq \tau_{k} \in \mathbb{R}$, and satisfies the difference equation at times $\tau_{k}$. Therefore, $\phi$ satisfies $\left(\mathbf{E}_{\lambda}\right)$ and, similarly, $\left(E_{\lambda}\right)$, and is therefore a complete solution, as claimed.

We conclude with a remark concerning the partial derivative in the variable $\phi$ of the operator $H$ at a given $\lambda \in \Lambda$ and reference solution $\phi \in B P C^{1}(\mathbb{R}, \Omega)^{\circ}$. Specifically, the partial derivative is

$$
D_{1} H(\phi, \lambda) \psi[t]=\psi(t)-\int_{-\infty}^{t} \mathbf{X}(t, s) D_{2} \mathbf{f}(s, \phi(s), \lambda) \psi(s) d s-\sum_{\tau_{k}<t} \mathbf{X}\left(t, \tau_{k}^{+}\right) D_{1} \mathbf{J}_{k}\left(\phi\left(\tau_{k}\right), \lambda\right) \psi\left(\tau_{k}\right) .
$$

If $\psi \in \mathcal{N}\left(D_{1} H(\phi, \lambda)\right)$, one can verify by taking derivatives of $D_{1} H(\phi, \lambda) \psi[t]=0$ that $\psi$ is a solution of the variational equation for $\left(\mathbf{E}_{\lambda}\right)$ about the solution $\phi$ :

$$
\begin{aligned}
\dot{\psi} & =\left[\mathbf{A}(t)+D_{2} \mathbf{f}(t, \phi(t), \lambda)\right] \psi, & t \neq \tau_{k} \\
\Delta \psi & =\left[\mathbf{B}_{k}+D_{1} \mathbf{J}_{k}\left(\phi\left(\tau_{k}, \lambda\right)\right] \psi,\right. & t=\tau_{k} .
\end{aligned}
$$

By construction, the above coincides with the variational equation for $\left(E_{\lambda}\right)$ about the reference solution $\phi$ :

$$
\begin{aligned}
\dot{\psi} & =\left[D_{2} f(t, \phi(t), \lambda) \psi, \quad t \neq \tau_{k}\right. \\
\Delta \psi & =D_{1} J_{k}\left(\phi\left(\tau_{k}, \lambda\right) \psi, \quad t=\tau_{k} .\right.
\end{aligned}
$$

\section{Fredholm properties of the linearized solution operator}

This section is organized as follows. First, we define bifurcation points and hyperbolicity of solutions. Following this, we prove that a lack of exponential dichotomy (nonhyperbolicity) always occurs at a bifurcation point. Then, at those parameter values $\lambda^{*}$ and solutions $\phi^{*}$ where a bifurcation could occur, we establish conditions under which the operator $D_{1} H\left(\phi^{*}, \lambda^{*}\right)$ is Fredholm, computing the index and characterizing various important subspaces of $B P C^{1}$ that will be relevant after.

\subsection{Bifurcation points}

The material in this section follows loosely the presentation of [Pötzsche, 2010]. We begin with a few definitions.

Definition 4.1. Let $\left(\phi^{*}, \lambda^{*}\right) \in B P C^{1} \times \Lambda$. We will say $\left(E_{\lambda}\right)$ bifurcates at $\lambda=\lambda^{*}$ along $\phi^{*}$, or simply $\phi^{*}$ bifurcates at $\lambda^{*}$, if there exist a sequence $\lambda_{n} \in \Lambda$ with $\lambda_{n} \rightarrow \lambda^{*}$ such that $\left(E_{\lambda_{n}}\right)$ has two distinct bounded complete solutions $\phi^{1}\left(\lambda_{n}\right)$ and $\phi^{2}\left(\lambda_{n}\right)$, satisfying

$$
\lim _{n \rightarrow \infty} \phi^{1}\left(\lambda_{n}\right)=\lim _{n \rightarrow \infty} \phi^{2}\left(\lambda_{n}\right)=\phi^{*},
$$

with convergence in $B P C^{1}$.

Definition 4.2. Given $\lambda^{*} \in \Lambda$, a bounded complete solution $\phi^{*}$ of $\left(E_{\lambda^{*}}\right)$ is hyperbolic if the variational equation $\left(V_{\lambda^{*}, \phi^{*}}\right)$ admits an exponential dichotomy on $\mathbb{R}$. Otherwise, $\phi^{*}$ is nonhyperbolic.

Proposition 3. Let hypotheses H1-H2 be satisfied. If a bounded complete solution $\phi^{*}$ bifurcates at $\lambda^{*}$, then $\phi^{*}$ is nonhyperbolic.

Proof. Choose a uniformly bounded pair $(\mathbf{A}, \mathbf{B})$ for which hypothesis H0 is satisfied, and define the operator $H$ with respect to the associated augmented system $\left(\mathbf{E}_{\lambda}\right)$. Then, for this system, hypotheses $\mathrm{H} 0-\mathrm{H} 2$ are satisfied and any statement regarding solutions of $\left(E_{\lambda}\right)$ apply to solutions of $\left(\mathbf{E}_{\lambda}\right)$ and vice versa. 
Proceeding by contrapositive, we consider the map $\mathcal{L}=D_{1} H\left(\phi^{*}, \lambda^{*}\right): B P C^{1} \rightarrow B P C^{1}$ and prove that if $\phi^{*}$ has an exponential dichotomy, then $\mathcal{L}$ is a Banach space isomorphism. Invoking the implicit function theorem will then demonstrate that $\phi^{*}$ does not bifurcate at $\lambda^{*}$.

By the bounded inverse theorem, it suffices to prove that $\mathcal{L}$ is a bounded bijection. Boundedness follows by Corollary 3.2. To begin, we show that $\mathcal{L}$ is injective. If $\mathcal{L} \psi=0$, then $\psi$ satisfies the variational equation $\left(V_{\phi^{*}, \lambda^{*}}\right)$. Thus, if $V(t, s)$ is the evolution operator of $\left(V_{\phi^{*}, \lambda^{*}}\right)$, then $\psi$ satisfies the equation $\psi(t)=V(t, s) \psi(s)$ for all $t \geq s$. By exponential dichotomy,

$$
P(t) \psi(t)=P(t) V(t, s) \psi(s)=V(t, s) P(s) \psi(s),
$$

which implies $|P(t) v(t)| \leq K e^{-\alpha(t-s)}|\psi(s)|$ for all $t \geq s$. Since $\psi$ is bounded, taking $s \rightarrow-\infty$, produces the bound $|P(t) \psi(t)| \leq 0$ for all $t \in \mathbb{R}$, which shows $\psi \in \mathcal{N}(P)$. Applying $V(s, t)=V^{-1}(t, s)$ to both sides of $\psi(t)=V(t, s) \psi(s)$ produces $\psi(s)=V(s, t) \psi(t)$, which holds for all $t \geq s$. Finally, since $\psi \in \mathcal{N}(P)$, we have $[I d-P] \psi=\psi$, and so

$$
\psi(s)=V(s, t) \psi(t)=V(s, t)[I d-P(t)] \psi(t),
$$

which implies $|\psi(s)| \leq K e^{\alpha(s-t)}|\psi(t)|$ for all $t \geq s$. With boundedness of $\psi$, taking the limit $t \rightarrow \infty$ produces the bound $|\psi(s)| \leq 0$, which holds for all $s \in \mathbb{R}$. Therefore, $\psi=0$, proving that $\mathcal{L}$ is injective.

We now prove that $\mathcal{L}$ is surjective. We must prove that for all $\psi \in B P C^{1}$, there exists $\gamma \in B P C^{1}$ such that $\mathcal{L} \gamma=\psi$. Since an element of $B P C^{1}$ is uniquely determined by its derivative in each interval $\left[\tau_{k}, \tau_{k+1}\right)$, its discontinuities at times $\tau_{k}$, and a given initial condition, it suffices to find a solution in $B P C^{1}$ of the impulsive differential equation

$$
\begin{aligned}
\gamma^{\prime}(t) & =\frac{d}{d t} L \gamma[t]+\psi^{\prime}(t), & & t \neq \tau_{k} \\
\Delta \gamma\left(\tau_{k}\right) & =\Delta L \gamma\left[\tau_{k}\right]+\Delta \psi\left(\tau_{k}\right) & & t=\tau_{k},
\end{aligned}
$$

where $\mathcal{L} \gamma=\gamma-L \gamma$ for the linear integral-sum operator $L$ appearing in (11). It can be verified that the above impulsive differential equation is equivalent to the following:

$$
\begin{aligned}
& \gamma^{\prime}=\left[\mathbf{A}(t)+D_{2} \mathbf{f}\left(t, \phi^{*}(t), \lambda\right)\right] \gamma+\left(\psi^{\prime}-\mathbf{A}(t) \psi\right), \quad t \neq \tau_{k} \\
& \Delta \gamma=\left[\mathbf{B}_{k}+D_{1} \mathbf{J}_{k}\left(\phi^{*}\left(\tau_{k}\right), \lambda^{*}\right)\right] \gamma+\left(\Delta \psi-\mathbf{B}_{k} \psi\right), \quad t=\tau_{k} .
\end{aligned}
$$

The associated homogeneous equation to (12) is precisely $\left(V_{\phi^{*}, \lambda^{*}}\right)$ which, by assumption, posesses an exponential dichotomy with exponent $\alpha$. Introduce the function

$$
\begin{aligned}
\gamma(t) & =\int_{0}^{\infty} G(t, s)\left[\psi^{\prime}(s)-\mathbf{A}(s) \psi(s)\right] d s+\sum_{j=-\infty}^{\infty} G\left(t, \tau_{j}^{+}\right)\left[\Delta \psi\left(\tau_{j}\right)-\mathbf{B}_{j} \psi\left(\tau_{j}\right)\right], \\
G(t, s) & = \begin{cases}V(t, s) P(s), & t>s \\
-V(t, s)[I d-P(s)], & t \leq s,\end{cases}
\end{aligned}
$$

whose definition is inspired by a similar construction appearing in [Kiskinov et. al, 2015]. Using the properties of exponential dichotomy, it is easily verified that $\gamma \in B P C^{1}$, and that $\gamma$ satisfies the impulsive differential equation (12). By the above discussion, $\gamma$ satisfies $T \gamma=\psi$. Therefore, $T=D_{1} H\left(\phi^{*}, \lambda^{*}\right)$ is a bounded linear bijection, and the proposition is proved.

\subsection{Fredholm properties of the linearized solution operator}

The main result of this section concerns the Fredholm index of the linearized solution operator, $D_{1} H\left(\phi^{*}, \lambda^{*}\right)$, when the variational equation $\left(V_{\phi^{*}, \lambda^{*}}\right)$ posesses exponential dichotomies on the half-lines $(-\infty, 0]$ and $[0, \infty)$. For full generality (and also to simplify the notation), we will present the result for an arbitrary linear impulsive system (L):

$$
\begin{aligned}
x^{\prime} & =L(t) x, \quad t \neq \tau_{k} \\
\Delta x & =M_{k} x, \quad t=\tau_{k},
\end{aligned}
$$


and the associated adjoint equation

$$
\begin{aligned}
y^{\prime} & =-L^{*}(t) y, & & t \neq \tau_{k} \\
\Delta y & =-\left[\left(I+M_{k}\right)^{-1}\right]^{*} M_{k}^{*} y, & & t=\tau_{k} .
\end{aligned}
$$

Consider the solution operator $\mathcal{L}: B P C^{1} \rightarrow B P C^{1}$ defined as follows:

$$
\mathcal{L} \psi=\psi-\int_{-\infty}^{t} \mathbf{X}(t, s)[L(s)-\mathbf{A}(s)] \psi(s) d s-\sum_{\tau_{k}<t} \mathbf{X}\left(t, \tau_{k}^{+}\right)\left[M_{k}-\mathbf{B}_{k}\right] \psi\left(\tau_{k}\right),
$$

where $\mathbf{X}(t, s)$ is, once again, the transition operator associated to (5). Note that if $(\mathrm{L})=\left(\mathbf{V}_{\lambda, \phi}\right)$, then $\mathcal{L}=D_{1} H\left(\phi^{*}, \lambda^{*}\right)$. We will derive conditions under which $\mathcal{L}$ is Fredholm, and we will compute the index. This will be done through a series of lemmas.

Lemma 2. Let $(\mathrm{L})$ be reversible and posess exponential dichotomies on $(-\infty, 0]$ and $[0-, \infty)$. If $g \in \operatorname{im} \mathcal{L}$, then for all bounded solutions $\psi \in B P C^{1}$ satisfying the adjoint equation $\left(\mathrm{L}^{*}\right)$, we have

$$
\left.\mathcal{F}(\psi, g):=\int_{-\infty}^{\infty}\langle\psi(t), \dot{g}(t)-\mathbf{A}(t) g(t)\rangle d t+\sum_{k \in \mathbb{Z}}\left\langle\psi\left(\tau_{k}^{+}\right), \Delta g\left(\tau_{k}\right)-\mathbf{B}_{k} g\left(\tau_{k}\right)\right]\right\rangle=0
$$

Proof. By Proposition 1, if (L) has exponential dichotomies on half-lines $(-\infty, 0]$ and $[0, \infty)$ with projectors $P_{-}$and $P_{+}$, then so does the adjoint equation $\left(\mathrm{L}^{*}\right)$, with projectors $I d-P_{-}^{*}$ and $I d-P_{+}^{*}$. The dichotomy properties then imply that if $\psi \in B P C^{1}$ solves the adjoint equation, then we must have $\psi(t) \rightarrow 0$ exponentially as $|t| \rightarrow \infty$.

Now, suppose $\mathcal{L} x=g$ for some $x \in B P C^{1}$. Differentiating both sides and examining discontinuities at impulse times, we find

$$
\begin{aligned}
\dot{x}(t)-L(t) x & =\dot{g}(t)-\mathbf{A}(t) g, & & t \neq \tau_{k} \\
\Delta x-M_{k} x & =\Delta g-\mathbf{B}_{k} g, & & t=\tau_{k} .
\end{aligned}
$$

With the above equalities at hand, we compute

$$
\begin{aligned}
\int_{-\infty}^{\infty}\langle\psi, \dot{g}-\mathbf{A} g\rangle d t & =\sum_{k \in \mathbb{Z}} \int_{\tau_{k}^{+}}^{\tau_{k+1}}\langle\psi, \dot{x}-L x\rangle d t \\
& =\sum_{k \in \mathbb{Z}} \int_{\tau_{k}^{+}}^{\tau_{k+1}}\langle\psi, \dot{x}\rangle-\left\langle L^{*} \psi, x\right\rangle d t \\
& =\sum_{k \in \mathbb{Z}} \int_{\tau_{k}^{+}}^{\tau_{k+1}}\langle\psi, \dot{x}\rangle+\langle\dot{\psi}, x\rangle d t \\
& =\sum_{k \in \mathbb{Z}} \int_{\tau_{k}^{+}}^{\tau_{k+1}} \frac{d}{d t}\langle\psi, x\rangle d t \\
& =\sum_{k \in \mathbb{Z}}\left\langle\psi\left(\tau_{k+1}\right), x\left(\tau_{k+1}\right)\right\rangle-\left\langle\psi\left(\tau_{k}^{+}\right), x\left(\tau_{k}^{+}\right)\right\rangle \\
\left\langle\psi\left(\tau_{k}\right), \Delta g\left(\tau_{k}\right)-\mathbf{B}_{k} f\left(\tau_{k}\right)\right\rangle & =\left\langle\psi\left(\tau_{k}\right), \Delta x\left(\tau_{k}\right)\right\rangle-\left\langle\psi\left(\tau_{k}\right), M_{k} x\left(\tau_{k}\right)\right\rangle \\
& =\left\langle\psi\left(\tau_{k}\right), \Delta x\left(\tau_{k}\right)\right\rangle+\left\langle\Delta \psi\left(\tau_{k}\right),\left(I+M_{k}\right) x\left(\tau_{k}\right)\right\rangle
\end{aligned}
$$

which implies

$$
\begin{aligned}
\left\langle\psi\left(\tau_{k}^{+}\right), \Delta f\left(\tau_{k}\right)-\mathbf{B}_{k} f\left(\tau_{k}\right)\right\rangle & =\left\langle\psi\left(\tau_{k}\right)+\Delta \psi\left(\tau_{k}\right), \Delta x\left(\tau_{k}\right)-M_{k} x\left(\tau_{k}\right)\right\rangle \\
& =\left\langle\Delta \psi\left(\tau_{k}\right)+\psi\left(\tau_{k}\right), \Delta x\left(\tau_{k}\right)\right\rangle+\left\langle\Delta \psi\left(\tau_{k}\right), x\left(\tau_{k}\right)\right\rangle \\
& =\left\langle\psi\left(\tau_{k}^{+}\right), \Delta x\left(\tau_{k}\right)\right\rangle+\left\langle\Delta \psi\left(\tau_{k}\right), x\left(\tau_{k}\right)\right\rangle \\
& =\left\langle\psi\left(\tau_{k}^{+}\right), x\left(\tau_{k}^{+}\right)\right\rangle-\left\langle\psi\left(\tau_{k}^{+}\right), x\left(\tau_{k}\right)\right\rangle+\left\langle\Delta \psi\left(\tau_{k}\right), x\left(\tau_{k}\right)\right\rangle \\
& =\left\langle\psi\left(\tau_{k}^{+}\right), x\left(\tau_{k}^{+}\right)\right\rangle-\left\langle\psi\left(\tau_{k}\right), x\left(\tau_{k}\right)\right\rangle
\end{aligned}
$$


Substituting (16) and (17) into (14), we have

$$
\begin{aligned}
\mathcal{F}(\psi, g) & =\sum_{k \in \mathbb{Z}}\left\langle\psi\left(\tau_{k+1}\right), x\left(\tau_{k+1}\right)\right\rangle-\left\langle\psi\left(\tau_{k}\right), x\left(\tau_{k}\right)\right\rangle \\
& =\lim _{k, j \rightarrow \infty}\left\langle\psi\left(\tau_{k}\right), x\left(\tau_{k}\right)\right\rangle-\left\langle\psi\left(\tau_{-j}\right), x\left(\tau_{-j}\right)\right\rangle .
\end{aligned}
$$

The above limit is zero because of the exponential decay of $\psi$, along with boundedness of $x$. Therefore, $\mathcal{F}(\psi, g)=0$, as claimed.

The following is a converse of the above lemma.

Lemma 3. Let $(\mathrm{L})$ be reversible and possess exponential dichotomies on $(-\infty, 0]$ and $[0, \infty)$. If for some $g \in B P C^{1}$, the operator $\mathcal{F}$ defined in (14) satisfies $\mathcal{F}(\psi, g)=0$ for all bounded solutions $\psi$ of the adjoint equation $\left(\mathrm{L}^{*}\right)$, then $g \in R(\mathcal{L})$.

Proof. Let $P$ and $Q$ denote the projection-valued operators for exponential dichotomies on $[0, \infty)$ and $(-\infty, 0]$ respectively. Denote $P_{0}=P(0), Q_{0}=Q(0)$, and similar for their adjoints. Since (L) is reversible and has Cauchy matrix $X(t, s)$, we have $X(t, s)=X(t, 0) X^{-1}(s, 0):=X(t) X^{-1}(s)$, and $Y(t):=V^{-1}(t)^{*}$ is a fundamental matrix solution of the adjoint equation. Without loss of generality, let $X(0)=I d$. Let $\eta \in \mathbb{R}^{n}$ satisfy $\eta^{*}\left(P_{0}-\left(I d-Q_{0}\right)\right)=0$, and consider the function

$$
\tilde{\psi}(t)=\left\{\begin{array}{lr}
Y(t)\left(I-P_{0}^{*}\right) \eta, & t \geq 0 \\
Y(t) Q_{0}^{*} \eta, & t<0
\end{array}\right.
$$

Recall that the adjoint equation to $\left(\mathrm{L}^{*}\right)$ has an exponential dichotomy on $[0, \infty)$ with projector $I-P^{*}$, and on $(-\infty, 0]$ with projector $I-Q^{*}$. From this, it follows that $\psi$ is bounded. Also, since $Q^{*} \eta=\left(I-P^{*}\right) \eta$ due to the definition of $\eta$, we have $\tilde{\psi}(t)=Y(t) Q^{*} \eta$ for all $t \in \mathbb{R}$, meaning that $\tilde{\psi}$ is a solution of the adjoint equation. Define

$$
G_{1}(s)= \begin{cases}Q_{0} X^{-1}(s), & s \leq 0 \\ \left(I d-P_{0}\right) X^{-1}(s), & s>0\end{cases}
$$

Notice that $\tilde{\psi}^{*}(t)=\eta^{*} G_{1}(t)$. Then, one finds

$$
\eta^{*}\left[\int_{-\infty}^{\infty} G_{1}(s)[\dot{g}(s)-\mathbf{A}(s) g(s)] d s+\sum_{k \in \mathbb{Z}} G_{1}\left(\tau_{k}^{+}\right)\left[\Delta g\left(\tau_{k}\right)-\mathbf{B}_{k} g\left(\tau_{k}\right)\right]\right]=\mathcal{F}(\tilde{\psi}, g),
$$

which, by assumption, is zero. By the Fredholm Alternative theorem, there exists $\xi \in X$ such that

$$
[P-(I d-Q)] \xi=\int_{-\infty}^{\infty} G_{1}(s)[\dot{g}(s)-\mathbf{A}(s) g(s)] d s+\sum_{k \in \mathbb{Z}} G_{1}\left(\tau_{k}^{+}\right)\left[\Delta g\left(\tau_{k}\right)-\mathbf{B}_{k} g\left(\tau_{k}\right)\right] .
$$

Assume without loss of generality that $\tau_{0}=0$. Define the following:

$$
\begin{aligned}
& x(t)=\left\{\begin{array}{l}
X(t) P \xi+\int_{0}^{\infty} G_{2}(t, s)[\dot{g}-\mathbf{A} g](s) d s+\sum_{k \geq 0} G_{2}\left(t, \tau_{k}^{+}\right)\left[\Delta g_{k}-\mathbf{B}_{k} g_{k}\right], \\
X(t)(I d-Q) \xi+\int_{-\infty}^{0} G_{2}(t, s)[\dot{g}-\mathbf{A} g](s) d s+\sum_{k<0} G_{2}\left(t, \tau_{k}^{+}\right)\left[\Delta g_{k}-\mathbf{B}_{k} g_{k}\right], t \leq 0,
\end{array}\right. \\
& G_{2}(t, s)= \begin{cases}X(t) P X^{-1}(s), & 0 \leq s \leq t \\
-X(t)(I d-P) X^{-1}(s), & 0 \leq t<s \\
X(t) Q X^{-1}(s), & s \leq t \leq 0 \\
-X(t)(I d-Q) X^{-1}(s), & t<s \leq 0 .\end{cases}
\end{aligned}
$$


It is readily verified that $x$ is a solution (15). Moreover, it is bounded. Indeed, if we set $h=\dot{g}-\mathbf{A} g$, then for for $t>0$ we have

$$
\begin{aligned}
\left|\int_{0}^{\infty} G_{2}(t, s) h(s) d s\right| & \leq\left|\int_{0}^{t} X(t) P X^{-1}(s) h(s) d s\right|+\left|\int_{t}^{\infty} X(t)(I d-P) X^{-1}(s) h(s) d s\right| \\
& =\left|\int_{0}^{t} P X(t, s) h(s) d s\right|+\left|\int_{t}^{\infty}(I d-P) X(t, s) h(s) d s\right| \\
& \leq \int_{0}^{t} K e^{-\alpha(t-s)}|h(s)| d s+\int_{t}^{\infty} K e^{\alpha(t-s)}|h(s)| d s \\
& \leq \frac{2 K}{\alpha}\|h\|_{\infty}<\infty .
\end{aligned}
$$

Similarly, one can show that the summation term is bounded for $t>0$, and that $x(t)$ is bounded for $t \leq 0$. Therefore, $x \in B P C^{1}$ and, in particular, $\mathcal{L} x=g$, so that $g \in \mathcal{R}(\mathcal{L})$, as required.

Theorem 2. Let $(\mathrm{L})$ be reversible and posess exponential dichotomies on $(-\infty, 0]$ and $[0-, \infty)$. The following are true.

- $g \in \mathcal{R}(\mathcal{L})$ if and only if $\mathcal{F}(\psi, g)=0$ for all $\psi \in B P C^{1}$ satisfying $\left(\mathrm{L}^{*}\right)$.

- The operator $\mathcal{L}$ is Fredholm.

If, in addition, the homogeneous equation (5) (with fundamental matrix $\mathbf{X}(t, s)$ ) posesses an exponential dichotomy on $\mathbb{R}$, then the index of $\mathcal{L}$ is

$$
\text { ind } \mathcal{L}=\operatorname{dim}(S \cap U)-\operatorname{dim}\left(S^{\perp} \cap U^{\perp}\right),
$$

where $S=\mathcal{R}\left(P_{0}\right), U=\mathcal{N}\left(Q_{0}\right)$, and $P$ is the projector on $[0, \infty)$ while $Q$ is the projector on $(-\infty, 0]$ for $(\mathrm{L}), P_{0}=P(0)$ and $Q_{0}=Q(0)$.

Proof. The first propositionerty characterizing $\mathcal{R}(\mathcal{L})$ follows by lemma 2 and lemma 3 . Therefore, we begin by showing that $\mathcal{R}(\mathcal{L})$ is closed and has finite codimension. To begin, notice that if $\psi$ is a bounded solution of the adjoint equation $\left(\mathrm{L}^{*}\right)$, then $\psi(0) \in S^{\perp} \cap U^{\perp}$; this can be seen as follows. First, it is clear that $S^{\perp}=\mathcal{R}\left(I-P_{0}^{*}\right)$ and $U^{\perp}=\mathcal{R}\left(Q_{0}^{*}\right)$. Then, by definition of exponential dichotomies, one can see that if $\psi_{0} \in S^{\perp} \cap U^{\perp}$, then $Y(t) \psi_{0}$ is a bounded solution of the adjoint equation $\left(\mathrm{L}^{*}\right)$, where $Y(t)=X^{*}(t)^{-1}$ is a fundamental matrix for the adjoint equation. In particular, all bounded solutions are of this form ${ }^{1}$. This establishes an isomorphism between the bounded solutions of $\left(\mathrm{L}^{*}\right)$ and $S^{\perp} \cap U^{\perp}$. We will now abuse notation and identify a bounded solution $\psi$ of the adjoint equation with an element of $S^{\perp} \cap U^{\perp}$ by said isormorphism. Define the linear map $\mathcal{F}: S^{\perp} \cap U^{\perp} \rightarrow\left(B P C^{1}\right)^{*}$ by

$$
\mathcal{F}(\psi)[f]=\mathcal{F}(\psi, f) .
$$

It follows that $\mathcal{F}\left(S^{\perp} \cap U^{\perp}\right)$ is finite-dimensional, and by the characterization of $\mathcal{R}(\mathcal{L})$, we have that $\mathcal{R}(\mathcal{L})$ is annihilated by some finite-dimensional subspace of $\mathcal{F}\left(S^{\perp} \cap U^{\perp}\right)$. Therefore, $\mathcal{R}(\mathcal{L})$ is closed and has finite codimension, so $\mathcal{L}$ is Fredholm.

Suppose (5) has an exponential dichotomy on $\mathbb{R}$. Let $\mathcal{F}(\psi)=0$ for some bounded solution of $\left(\mathrm{L}^{*}\right)$. Then, the same proof as in Proposition 3 guarantees the existence of a unique $\mu \in B P C^{1}$ such that

$$
\begin{aligned}
\dot{\mu}-\mathbf{A}(t) \mu & =\psi, & & t \neq \tau_{k} \\
\Delta \mu-\mathbf{B}_{k} \mu\left(\tau_{k}\right) & =\psi\left(\tau_{k}^{+}\right), & & t=\tau_{k} .
\end{aligned}
$$

But then, we have

$$
\mathcal{F}[\psi](\mu)=\int_{-\infty}^{\infty}\langle\psi, \psi\rangle d t+\sum_{k \in \mathbb{Z}}\left\langle\psi\left(\tau_{k}^{+}\right), \psi\left(\tau_{k}^{+}\right)\right\rangle=0
$$

\footnotetext{
${ }^{1}$ This can be seen by noticing that exponential dichotomy with projection-valued operator $P$ for the fundamental matrix $X$ implies inequalities of the form $|X(t) P(0) \xi| \leq K e^{-\alpha(t-s)}|X(s) P(0) \xi|$ for all $s \leq t$ and $\xi \in X$.
} 
which implies that $\psi=0$, so $\mathcal{F}$ is injective, defining an isomorphism onto its range. Consequently, $\mathcal{R}(\mathcal{F}) \sim$ $S^{\perp} \cap U^{\perp}$ annihilates $\mathcal{R}(\mathcal{L})$, and we obtain that

$$
\operatorname{codim} \mathcal{R}(\mathcal{L})=\operatorname{dim} \mathcal{R}(\mathcal{F})=\operatorname{dim} S^{\perp} \cap U^{\perp} .
$$

By definition, the index of $\mathcal{L}$ is $\operatorname{dim} \mathcal{N}(\mathcal{L})-\operatorname{codim} \mathcal{R}(\mathcal{L})$. The rest of the proof follows the same lines as the proof of the orthogonality theorem appearing in [Palmer, 1984] and is omitted.

Note that assumption $\mathrm{H} 0$ implies that (5) posesses an exponential dichotomy on $\mathbb{R}$ with projector $P=I$, so the final assumption of Theorem 2 concerning $\mathbf{X}(t, s)$ holds whenever $H 0$ does, if one is considering $(\mathrm{L})=\left(V_{\lambda, \phi}\right)$. With the above theorem, we now state the appropositionriate result for the operator $D_{1} H\left(\phi^{*}, \lambda^{*}\right)$. First, some additional assumptions.

H3. Let $q, r \in \mathbb{N}$ and $\lambda^{*} \in \Lambda$ be given. $\left(E_{\lambda^{*}}\right)$ admits a complete permanent ${ }^{2}$ solution $\phi^{*} \in B P C^{1}(\mathbb{R}, \Omega)$ such that the variational equation $\left(V_{\lambda^{*}, \phi^{*}}\right)$ admits an exponential dichotomy on both $[0, \infty)$ and $(-\infty, 0]$ with respective projectors $P$ and $Q$ satisfying

$$
\begin{aligned}
\mathcal{R}\left(P_{0}\right) \cap \mathcal{N}\left(Q_{0}\right) & =\operatorname{span}\left\{\xi_{1}, \ldots, \xi_{p}\right\} \\
\left(\mathcal{R}\left(P_{0}\right) \oplus \mathcal{N}\left(Q_{0}\right)\right)^{\perp} & =\operatorname{span}\left\{\xi_{1}^{\prime}, \ldots, \xi_{r}^{\prime}\right\}
\end{aligned}
$$

and linearly ndependent vectors $\xi_{1}, \ldots, \xi_{q} \in X$ and $\xi_{1}^{\prime}, \ldots, \xi_{r}^{\prime} \in X^{\prime}$.

H4. $\left(V_{\lambda^{*}, \phi^{*}}\right)$ is reversible.

Proposition 4. If (H1)-(H6) hold, the linear operator $D_{1} H\left(\phi^{*}, \lambda^{*}\right): B P C^{1} \rightarrow B P C^{1}$ is Fredholm of index $p-r$ and one has, up to isomorphism,

$$
\begin{aligned}
\mathcal{N}\left(D_{1} H\left(\phi^{*}, \lambda^{*}\right)\right) & =\operatorname{span}\left\{V_{\lambda^{*}} \xi_{1}, \ldots, V_{\lambda^{*}} \xi_{p}\right\} \\
\mathcal{N}\left(D_{1} H\left(\phi^{*}, \lambda^{*}\right)^{\prime}\right) & \sim \operatorname{span}\left\{Y_{\lambda^{*}} \xi_{1}^{\prime}, \ldots, Y_{\lambda^{*}} \xi_{r}^{\prime}\right\} \\
\mathcal{N}\left(D_{1} H\left(\phi^{*}, \lambda^{*}\right)^{\prime}\right) & =\operatorname{span}\left\{\mathcal{F}\left[Y_{\lambda^{*}} \xi_{1}^{\prime}\right], \ldots, \mathcal{F}\left[Y_{\lambda^{*}} \xi_{r}^{\prime}\right]\right\}
\end{aligned}
$$

where $V_{\lambda^{*}}$ is the fundamental matrix solution of $\left(V_{\lambda^{*}, \phi^{*}}\right)$ satisfying $V_{\lambda^{*}}(0)=I, Y_{\lambda^{*}}=\left(V_{\lambda^{*}}^{-1}\right)^{\prime}$ is the fundamental matrix of the adjoint system to $\left(V_{\lambda^{*}, \phi^{*}}\right)$, and $\mathcal{F}$ is as defined in (18). The elements of the above spans are linearly independent.

Proof. That $\mathcal{L}=D_{1} H\left(\phi^{*}, \lambda^{*}\right)$ is Fredholm under assumptions (H1)-(H6) follows directly by Theorem 2. As for the index, denote $S=\mathcal{R}\left(P_{0}\right)$ and $U=\mathcal{N}\left(Q_{0}\right)$ as in the proof of Theorem 2. By assumption (H5), we have

$$
\operatorname{dim}(S \cap U)=\operatorname{dim}\left(\mathcal{R}\left(P_{0}\right) \cap \mathcal{N}\left(Q_{0}\right)\right)=p
$$

and also,

$$
\begin{aligned}
\operatorname{dim}\left(S^{\perp} \cap U^{\perp}\right) & =\operatorname{dim}\left(\left[\left[S^{\perp} \cap U^{\perp}\right]^{\perp}\right]^{\perp}\right) \\
& =\operatorname{dim}\left(\left[\left(S^{\perp}\right)^{\perp} \oplus\left(U^{\perp}\right)^{\perp}\right]^{\perp}\right) \\
& =\operatorname{dim}\left([S \oplus U]^{\perp}\right) \\
& =\operatorname{dim}\left(\left[\mathcal{R}\left(P_{0}\right) \oplus \mathcal{N}\left(Q_{0}\right)\right]^{\perp}\right) \\
& =r
\end{aligned}
$$

so that ind $(\mathcal{L})=p-r$. The assertions concerning $(19)$ are proven as follows. Recall that $\mathcal{N}(\mathcal{L})$ consists of all bounded solutions of the variational equation $\left(V_{\lambda^{*}, \phi^{*}}\right)$. Any bounded solution $y=V_{\lambda^{*}} y(0)$ of the variational equation must have an initial condition $y(0) \in S \cap U^{3}$. By hypothesis (H5), $y(0) \in \operatorname{span}\left\{\xi_{1}, \ldots, \xi_{p}\right\}$, and since the $\xi_{i}$ are linearly independent, the first equality of (19) is proven.

\footnotetext{
$\left.\overline{{ }^{2} \phi \in B P C^{1}(\mathbb{R}, \Omega) \text { is permanent if } \inf _{t \in \mathbb{R}} \operatorname{dist}(\phi(t)}, \partial \Omega\right)>0$.

${ }^{3}$ The proof of this assertion is essentially the same as the proof that a bounded solution of the adjoint equation must have an initial condition on $S^{\perp} \cap U^{\perp}$; see the proof of Theorem 2 .
} 
For the second and third equalities, recall that we have $\mathcal{N}\left(\mathcal{L}^{\prime}\right)=\mathcal{R}(\mathcal{L})^{\perp}$. By the proof of Theorem 2,

$$
\mathcal{R}(\mathcal{L})^{\perp}=\mathcal{R}(\mathcal{F})=\mathcal{F}\left(S^{\perp} \cap U^{\perp}\right) \sim S^{\perp} \cap U^{\perp}=(S \oplus U)^{\perp},
$$

and since $S^{\perp} \cap U^{\perp}$ is isomorphic to the space of bounded solutions of $\left(\mathrm{L}^{*}\right)$ (in this case, the adjoint equation associated to the variational equation), we obtain the result claimed.

\subsection{Construction of Lyapunov-Schmidt Projectors}

With Theorem 4 at hand, we will construct the Lyapunov-Schmidt projectors associated to the Fredholm operator $\mathcal{L}=D_{1} H\left(\phi^{*}, \lambda^{*}\right)$. First, define $\mathcal{P} \in L\left(B P C^{1}\right)$ by

$$
\mathcal{P} x=\sum_{i=1}^{p}\left\langle\eta_{i}^{\prime}, x(0)\right\rangle V_{\lambda^{*}} \xi_{i},
$$

where $\eta_{1}^{\prime}, \ldots, \eta_{q}^{\prime} \in X^{\prime}$ are such that $\left\langle\eta_{i}^{\prime}, \xi_{j}\right\rangle=\delta_{i, j}$ for $1 \leq i, j \leq q$. By construction, $\mathcal{P}$ is a bounded projection onto $\mathcal{N}(\mathcal{L})$. For the projection $\mathcal{Q} \in L\left(B P C^{1}\right)$ onto $\mathcal{R}(\mathcal{L})$, the construction is a bit more complicated, and we require a few lemmas.

Lemma 4. Let $\omega \in B P C^{1}(0, \infty)$ be a function such that $\omega V_{\lambda^{*}}^{\prime}$ is bounded and normalized, in the sense that

$$
\int_{\mathbb{R}} \omega(s) d s+\sum_{k \in \mathbb{Z}} \omega\left(\tau_{k}\right)=1 .
$$

Then, there exist $f_{1}, \ldots, f_{r} \in B P C^{1}$ such that $\mathcal{F}\left[Y_{\lambda^{*}} \xi_{i}^{\prime}\right]\left(f_{j}\right)=\delta_{i, j}$ for $1 \leq i, j \leq r$. Specifically, $f_{j}$ is a solution of the impulsive differential equation

$$
\begin{aligned}
\dot{f}_{j}-A(t) f_{j} & =\omega V_{\lambda^{*}}^{\prime} \eta_{j}, & & t \neq \tau_{k} \\
\Delta f_{j}-\mathbf{B}_{k} f_{j}\left(\tau_{k}\right) & =\omega\left(\tau_{k}\right) V_{\lambda^{*}}^{\prime}\left(\tau_{k}^{+}\right) \eta_{j}, & & t=\tau_{k},
\end{aligned}
$$

where the $\eta_{j}$ are chosen so that $\left\langle\xi_{i}^{\prime}, \eta_{j}\right\rangle=\delta_{i j}$.

Proof. By assumption, since $\omega V_{\lambda^{*}}^{\prime}$ is bounded, exponential dichotomy of the homogeneous equation (5) guarantees the existence of functions $f_{j} \in B P C^{1}$ satisfying the impulsive differential equation given in the statement of the lemma. Then, by definition of $\mathcal{F}$, the vectors $\eta_{j}$ and duality, we have

$$
\begin{aligned}
\mathcal{F}\left[Y_{\lambda^{*}} \xi_{i}^{\prime}\right]\left(f_{j}\right) & =\int_{\mathbb{R}}\left\langle Y_{\lambda^{*}} \xi_{i}^{\prime}, V_{\lambda^{*}}^{\prime} \eta_{j} \omega\right\rangle d s+\sum_{k \in \mathbb{Z}}\left\langle Y_{\lambda^{*}}\left(\tau_{k}^{+}\right) \xi_{i}^{\prime}, V_{\lambda^{*}}^{\prime}\left(\tau_{k}^{+}\right) \eta_{j} \omega\left(\tau_{k}\right)\right\rangle \\
& =\int_{\mathbb{R}}\left\langle Y_{\lambda^{*}} V_{\lambda^{*}} \xi_{i}^{\prime}, \eta_{j}\right\rangle \omega d s+\sum_{k \in \mathbb{Z}}\left\langle Y_{\lambda^{*}}\left(\tau_{k}^{+}\right) V_{\lambda^{*}}\left(\tau_{k}^{+}\right) \xi_{i}^{\prime}, \eta_{j}\right\rangle \omega\left(\tau_{k}\right) \\
& =\int_{\mathbb{R}}\left\langle\xi_{i}^{\prime}, \eta_{j}\right\rangle \omega d s+\sum_{k \in \mathbb{Z}}\left\langle\xi_{i}^{\prime}, \eta_{j}\right\rangle \omega\left(\tau_{k}\right) \\
& =\delta_{i j} .
\end{aligned}
$$

Remark 4.1. There exist functions $\omega$ satisfying the conditions of the above lemma as follows. We construct one in the following. Identify $\left(V_{\lambda, \phi}\right)$ with (L) for brevity. By the assumptions of the lemma, we have with $\|L(t)\| \leq c$ for some $c \geq 0$, and so

$$
\left|V_{\lambda^{*}}(t)\right| \leq e^{c t+\sum_{\tau_{k} \in[0, t)} \log \left|I d+M_{k}\right|-\sum_{\tau_{j} \in(t, 0]} \log \left|I d+M_{j}\right|} \leq e^{c|t|+g(t)},
$$

where $g$, defined by

$$
g(t)=\sum_{\tau_{k} \in[-|t|,|t|]}|\log | I d+M_{k}||,
$$


is even, nondecreasing, nonnegative, and is an element of $B P C^{1}(\mathbb{R})$. In particular, there exists $g_{1} \in$ $B P C^{1}(\mathbb{R})$ such that $g \leq g_{1}$ and $e^{-g_{1}(t)}$ is integrable. Define $\omega_{1}(t)=\exp \left(-2 c|t|-g_{1}(t)\right)$. Then $\omega_{1} \in$ $B P C^{1}(0, \infty)$ is integrable (hence summable) and $\omega_{1} V_{\lambda^{*}}$ is clearly bounded. An obvious normalization produces the desired result.

With the above two lemmas in mind, the following is obvious.

Proposition 5. The operator $\mathcal{Q} \in L\left(B P C^{1}\right)$ defined by

$$
\mathcal{Q} y=y-\sum_{i=1}^{r} \mathcal{F}\left[Y_{\lambda^{*}} \xi_{i}^{\prime}\right](y) f_{i}
$$

is a projection onto $\mathcal{R}(\mathcal{L})$, provided the functions $f_{1}, \ldots, f_{r}$ are as given in Lemma 4

\section{Bifurcation theorems}

The results on this section are based on techniques from bifurcation theory in general Banach spaces. There are a wealth of references on this topic, including [Chow \& Hale, 1996; Kielhofer, 2012; Zeidler, 1993]. For our purposes, we will refer to the convenient appendix appearing in the article [Pötzsche, 2010]. Section 5.1 provides the abstract branching equation associated to $\left(E_{\lambda}\right)$, as well as some supplemental results that will be useful in Section 5.2, where sufficient conditons for bifurcations of bounded solutions are given.

\subsection{Abstract branching equation and supplemental results}

With the projectors of Section 4.3, together with our Fredholm theory for the opertor $D_{1} H\left(\phi^{*}, \lambda^{*}\right)$, we are ready to perform Lyapunov-Schmidt reduction.

Theorem 3 [Branching Equations]. Suppose H0-H4 hold. There exist open convex neighbourhoods $S \subseteq \mathbb{R}^{p}$ of $0, \lambda_{0} \subseteq \Lambda$ of $\lambda^{*}$ and a $C^{m}$ fuction $v: S \times \Lambda_{0} \rightarrow B P C^{1}$ satisfying $v\left(0, \lambda^{*}\right)=0$, $\operatorname{det} D_{1} v\left(0, \lambda^{*}\right)=0$, and

$$
\begin{aligned}
& H\left(\phi^{*}+\sum_{i=1}^{p} V_{\lambda^{*}} s_{i} \xi_{i}+v(s, \lambda), \lambda\right)-\sum_{j=1}^{r}\left[\int _ { \mathbb { R } } \left\langleY_{\lambda^{*}} \xi_{j}^{\prime}, \mathbf{f}\left(t, \phi^{*}, \lambda^{*}\right)+\dot{v}(s, \lambda)(t)-\mathbf{A}(t) v(s, \lambda)(t)\right.\right. \\
& \left.+D_{2} \mathbf{f}\left(t, \phi^{*}, \lambda^{*}\right) V_{\lambda^{*}} \sum_{i=1}^{p} \xi_{i} s_{i}\right\rangle d t+\sum_{k \in \mathbb{Z}}\left\langle Y_{\lambda^{*}}\left(\tau_{k}^{+}\right) \xi_{j}^{\prime}, \mathbf{J}_{k}\left(\phi^{*}, \lambda^{*}\right)+\left[\Delta v(s, \lambda)\left(\tau_{k}\right)-\mathbf{B}_{k} v(s, \lambda)\left(\tau_{k}\right)\right]\right. \\
& \left.\left.+D_{1} \mathbf{J}_{k}\left(\phi^{*}, \lambda^{*}\right) V_{\lambda^{*}}\left(\tau_{k}\right) \sum_{i=1}^{p} \xi_{i} s_{i}\right\rangle\right] f_{j}=0,
\end{aligned}
$$

for all $(s, \lambda) \in S \times \Lambda_{0}$, where the functions $f_{j}$ are solutions of (21) for an arbitrary normalization factor $\omega$. In particular, if there exists $s \in S$ satisfying the equation $g(s, \lambda)=0$, where $g: S \times \Lambda_{0} \rightarrow \mathbb{R}$ has components

$$
\begin{aligned}
g_{j}(s, \lambda) & =\int_{\mathbb{R}}\left\langle Y_{\lambda^{*}} \xi_{j}^{\prime}, \mathbf{f}\left(t, \phi^{*}, \lambda^{*}\right)-\mathbf{f}\left(t, \phi^{*}+V_{\lambda^{*}} \sum_{i=1}^{p} s_{i} \xi_{i}+v(s, \lambda), \lambda\right)+\dot{v}(s, \lambda)-\mathbf{A}(t) v(s, \lambda)\right. \\
& \left.+D_{2} \mathbf{f}\left(t, \phi^{*}+V_{\lambda^{*}} \sum_{i=1}^{p} s_{i} \xi_{i}+v(s, \lambda), \lambda\right) V_{\lambda^{*}} \sum_{i=1}^{p} s_{i} \xi_{i}\right\rangle+\sum_{k \in \mathbb{Z}}\left\langle Y_{\lambda^{*}}\left(\tau_{k}^{+}\right) \xi_{j}^{\prime}, \mathbf{J}_{k}\left(\phi^{*}, \lambda^{*}\right)\right. \\
& -\mathbf{J}_{k}\left(\phi^{*}+V_{\lambda^{*}} \sum_{i=1}^{p} s_{i} \xi_{i}+v(s, \lambda), \lambda\right)+\Delta v(s, \lambda)\left(\tau_{k}\right)-\mathbf{B}_{k} v(s, \lambda)\left(\tau_{k}\right) \\
& \left.+D_{1} \mathbf{J}_{k}\left(\phi^{*}+V_{\lambda^{*}} \sum_{i=1}^{p} s_{i} \xi_{i}+v(s, \lambda), \lambda\right) V_{\lambda^{*}}\left(\tau_{k}\right) \sum_{i=1}^{p} \xi_{i} s_{i}\right\rangle
\end{aligned}
$$

then $\phi=\phi^{*}+V_{\lambda^{*}} \sum_{i=1}^{p} s_{i} \xi_{i}+v(s, \lambda)$ satisfies $H(\phi, \lambda)=0$. 
Proof. The proof is a consequence of applying the result on branching equations from Lemma A.1 of [Pötzsche, 2010] together with our preparations on the Fredholm theory for $\mathcal{L}=D_{1} H\left(\phi^{*}, \lambda^{*}\right)$ of Section 4.2 and the Lyapunov-Schmidt projectors of Section 4.3. One must also keep in mind that the vectors $f_{j}$ for $1 \leq j \leq r$ given by Lemma 4 satisfy $(I-\mathcal{Q}) f_{j}=f_{j}$ and are linearly independent, while $\operatorname{dim} \mathcal{R}(I-\mathcal{Q})=$ $\operatorname{codim} \mathcal{R}(\mathcal{L})=r$, so we can choose $\mathcal{R}(I-\mathcal{Q})=\operatorname{span}\left\{f_{i}, \ldots, f_{r}\right\}$.

Notice that the branching equations depend explicitly on the choice of augmenting pair $(\mathbf{A}, \mathbf{B})$. The bifurcation conditions of the following section, however, will be independent of such a choice. Before moving to these results, we introduce linear functionals that will be useful later.

Lemma 5. If HO-H4 hold, the linear functionals $\mu_{i}: B P C^{1} \rightarrow \mathbb{R}$ for $1 \leq i \leq r$ defined by

$$
\mu_{i}(\phi)=\mathcal{F}\left[Y_{\lambda^{*}} \xi_{1}^{\prime}\right](\phi)=\int_{\mathbb{R}}\left\langle Y_{\lambda^{*}} \xi_{i}^{\prime}, \dot{\phi}-\mathbf{A} \phi\right\rangle d s+\sum_{k \in \mathbb{Z}}\left\langle Y_{\lambda^{*}}\left(\tau_{k}^{+}\right) \xi_{i}^{\prime}, \Delta \phi\left(\tau_{k}\right)-\mathbf{B}_{k} \phi\left(\tau_{k}\right)\right\rangle
$$

are continuous, and one has $\mathcal{R}\left(D_{1} H\left(0, \lambda^{*}\right)=\bigcap_{i=1}^{r} \mathcal{N}(\mu)\right.$.

Proof. By definition,

$$
\xi_{i}^{\prime} \in\left(\mathcal{R}\left(P_{0}\right) \oplus \mathcal{N}\left(Q_{0}\right)\right)^{\perp}=\mathcal{N}\left(I-P_{0}^{*}\right) \cap \mathcal{R}\left(I-Q_{0}^{*}\right),
$$

which implies both $P_{0}^{*} \xi_{i}^{\prime}=\xi_{i}^{\prime}$ and $\left(I-Q_{0}^{*}\right) \xi_{i}^{\prime}=\xi_{i}^{\prime}$. Therefore,

$$
\begin{aligned}
\int_{\mathbb{R}}\left\langle Y_{\lambda^{*}} \xi_{i}^{\prime}, \dot{\phi}-\mathbf{A} \phi\right\rangle d s & =\int_{-\infty}^{0}\left\langle Y_{\lambda^{*}} P^{\prime} \xi_{i}^{\prime}, \dot{\phi}-\mathbf{A} \phi\right\rangle d s+\int_{0}^{\infty}\left\langle Y_{\lambda^{*}}\left(I-Q^{*}\right) \xi_{i}^{\prime}, \dot{\phi}-\mathbf{A} \phi\right\rangle d s \\
& \leq\left|\xi_{i}^{\prime}\right| \cdot\|\dot{\phi}-\mathbf{A} \phi\|_{0}\left(\int_{-\infty}^{0}\left|Y_{\lambda^{*}}(t, 0) P^{*}(0)\right| d t+\int_{0}^{\infty}\left|Y_{\lambda^{*}}(0, t)\left(I-Q^{*}(t)\right)\right| d t\right) \\
& \leq\left(\left|\xi_{i}^{\prime}\right|(|1+\| \mathbf{A}| \mid) K \int_{\mathbb{R}} e^{-\alpha|t|} d t\right) \cdot\|\phi\|_{1} .
\end{aligned}
$$

An analogous bound is available for the summation term in (25). Therefore, $\mu_{i}$ is bounded. The assertion involving the range of $D_{1} H\left(0, \lambda^{*}\right)$ follows directly from Theorem 2 and Proposition 4.

We will also require a result concerning the time derivatives of the various derivatives of the operator $H$. The following lemma follows by direct computations and the proof is omitted.

Lemma 6. Let hypotheses $\mathrm{HO}_{-} \mathrm{H}_{4}$ be satisfied. Let $V^{u}=H^{u}\left(\phi^{*}, \lambda^{*}\right)$, where $u \leq m$ is either an integer or 2-index. Then,

$$
\begin{aligned}
\dot{V}^{u}-\mathbf{A} V^{u} & =\frac{d}{d t}\left[D^{u} \pi_{1}\left(\phi^{*}, \lambda^{*}\right)\right]-D^{u}\left[\mathbf{A}(t) \phi^{*}+\mathbf{f}\left(t, \phi^{*}, \lambda^{*}\right)\right], \\
\Delta V^{u}\left(\tau_{k}\right)-\mathbf{B}_{k} V^{u}\left(\tau_{k}\right) & =\Delta\left[D^{u} \pi_{1}\left(\phi^{*}, \lambda^{*}\right) \phi^{*}\left(\tau_{k}\right)\right]-D^{u}\left[\mathbf{B}_{k} \phi^{*}+\mathbf{J}_{k}\left(\phi^{*}, \lambda^{*}\right)\right],
\end{aligned}
$$

where $\pi_{1}: B P C^{1} \times \Lambda \rightarrow B P C^{1}$ is the projection $\pi_{1}(\phi, \lambda)=\phi$.

\subsection{Explicit bifurcation conditions}

In the following theorems, we will at times assume a trivial solution branch is known. That is, the following additional hypothesis is introduced.

H5. $f(t, 0, \lambda)=0$ on $\mathbb{R} \times \Lambda$ and $J_{k}(0, \lambda)=0$ on $\mathbb{Z} \times \Lambda$.

Remark 5.1. Note that if $\phi^{*}$ is a given solution independent on the parameter $\lambda$, the time-dependent change of variables $x=\phi^{*}+y$ produces an equivalent impulsive differential equation in the variable $y$ that satisfies H5. 
Theorem 4 [Bifurcation with an odd-dimensional kernel]. Let $\Lambda \subseteq \mathbb{R}$ and $m \geq 2$. If H1-H5 hold with $p=r$, then the trivial solution of $\left(E_{\lambda}\right)$ bifurcates at $\lambda^{*}$, provided $p$ is odd and the following transversality condition holds:

$$
\operatorname{det}\left(\int_{\mathbb{R}}\left\langle Y_{\lambda^{*}}(t) \xi_{j}^{\prime}, D_{2} D_{3} f\left(t, 0, \lambda^{*}\right) V_{\lambda^{*}}(t) \xi_{i}\right\rangle d t+\sum_{k \in \mathbb{Z}}\left\langle Y_{\lambda^{*}}\left(\tau_{k}^{+}\right) \xi_{j}^{\prime}, D_{1} D_{2} J_{k}\left(0, \lambda^{*}\right) V_{\lambda^{*}}\left(\tau_{k}\right) \xi_{i}\right\rangle\right)_{1 \leq i, j \leq p} \neq 0 .
$$

Proof. Choose a pair $(\mathbf{A}, \mathbf{B})$ such that condition H0 is satisfied for $\mathbf{X}(t, s)$, and define the solution operator $H$ for the equivalent augmented system $\left(\mathbf{E}_{\lambda}\right)$. We will use Theorem A.5 of [Pötzsche, 2010]. Let $\theta \in$ $\mathcal{N} D_{1} H\left(0, \lambda^{*}\right)$; it follows by Proposition 4 that $\theta=\sum_{j=1}^{p} s_{j} V_{\lambda^{*}} \xi_{j}$ for $s_{j} \in \mathbb{R}$. By Theorem 2 , we have $D_{1} D_{2} H\left(0, \lambda^{*}\right) \theta \in \mathcal{R} D_{1} H\left(0, \lambda^{*}\right)$ if and only if $\mathcal{F}\left[Y_{\lambda^{*}} \xi_{i}^{\prime}\right]\left(D_{1} D_{2} H\left(0, \lambda^{*}\right) \theta\right)=0$ for all $i=1, \ldots, p$. However, using Lemma 6 , this is seen to be equivalent to

$$
\sum_{j=1}^{p} s_{j}\left(\int_{\mathbb{R}}\left\langle Y_{\lambda^{*}} \xi_{i}^{\prime}, D_{2} D_{3} f\left(t, 0, \lambda^{*}\right) V_{\lambda^{*}} \xi_{j}\right\rangle d t+\sum_{k \in \mathbb{Z}}\left\langle Y_{\lambda^{*}}\left(\tau_{k}^{+}\right) \xi_{i}^{\prime}, D_{1} D_{2} J_{k}\left(0, \lambda^{*}\right) V_{\lambda^{*}}\left(\tau_{k}\right) \xi_{j}\right\rangle\right)=0
$$

for $i=1, \ldots, p$. The above defines a homogeneous $p \times p$ system of linear equations, and the determinant condition of the theorem guarantees it has a unique solution, from which we conclude that $s_{1}=\cdots=s_{p}=0$. Therefore, $\theta=0$, so that

$$
D_{1} D_{2} H\left(0, \lambda^{*}\right) \mathcal{N}\left(D_{1} H\left(0, \lambda^{*}\right)\right) \cap \mathcal{R}\left(D_{1} H\left(0, \lambda^{*}\right)\right)=\{0\}
$$

as required.

Theorem 5 [Fold bifurcation]. Let $\Lambda \subseteq \mathbb{R}$. Suppose H1-H5 hold with $p=r=1$. If

$$
g_{01}:=\int_{\mathbb{R}}\left\langle Y_{\lambda^{*}} \xi_{i}^{\prime}, D_{3} f\left(t, \phi^{*}, \lambda^{*}\right)\right\rangle d t+\sum_{k \in \mathbb{Z}}\left\langle Y_{\lambda^{*}}\left(\tau_{k}^{+}\right) \xi_{i}^{\prime}, D_{2} J_{k}\left(\phi^{*}, \lambda^{*}\right)\right\rangle \neq 0,
$$

then there exists $\rho>0$ and open convex neighbourhoods $U \subseteq B P C^{1}(\Omega)$ of $\phi^{*}, \lambda_{0} \subseteq \Lambda$ of $\lambda^{*}$, and $C^{m}$ functions $\phi:(-\rho, \rho) \rightarrow U, \lambda:(-\rho, \rho) \rightarrow \Lambda_{0}$ such that

- $\phi(0)=\phi^{*}, \lambda(0)=\lambda^{*}$, and $\dot{\phi}=V_{\lambda^{*}} \xi_{i}, \dot{\lambda}(0)=0$,

- each $\phi(s)$ is a complete solution of $\left(E_{\lambda(s)}\right)$ in $B P C^{1}(\Omega)$.

Moreover, in case $m \geq 2$ and under the additional assumption

$$
g_{20}:=\int_{\mathbb{R}}\left\langle Y_{\lambda^{*}} \xi_{i}^{\prime}, D_{2}^{2} f\left(t, \phi^{*}, \lambda^{*}\right)\left[V_{\lambda^{*}} \xi_{1}\right]^{2}\right\rangle d t+\sum_{k \in \mathbb{Z}}\left\langle Y_{\lambda^{*}}\left(\tau_{k}^{+}\right) \xi_{i}^{\prime}, D_{1}^{2} J_{k}\left(\phi^{*}, \lambda^{*}\right)\left[V_{\lambda^{*}}\left(\tau_{k}^{+}\right) \xi_{1}\right]^{2}\right\rangle \neq 0,
$$

$\phi^{*}$ bifurcates at $\lambda^{*}$, one has $\ddot{\lambda}(0)=-\frac{g_{20}}{g_{01}}$, and the following holds locally in $\Lambda_{0}$.

- Subcritical case: If $g_{20} / g_{01}>0$, there exists $\Omega_{0} \subseteq \Omega$ open and containing the image of $\phi^{*}$ such that then $\left(E_{\lambda}\right)$ has no complete solution in $B P C\left(\Omega_{0}\right)$ for $\lambda>\lambda^{*}, \phi^{*}$ is the unique complete solution of $\left(E_{\lambda^{*}}\right)$ in $B P C\left(\Omega_{0}\right)$, and there are exactly two distinct complete solutions in $B P C\left(\Omega_{0}\right)$ for $\lambda<\lambda^{*}$.

- Supercritical case: If $g_{20} / g_{01}>0$, there exists $\Omega_{0} \subseteq \Omega$ open and containing the image of $\phi^{*}$ such that then $\left(E_{\lambda}\right)$ has no complete solution in $B P C\left(\Omega_{0}\right)$ for $\lambda<\lambda^{*}, \phi^{*}$ is the unique complete solution of $\left(E_{\lambda^{*}}\right)$ in $B P C\left(\Omega_{0}\right)$, and there are exactly two distinct complete solutions in $B P C\left(\Omega_{0}\right)$ for $\lambda>\lambda^{*}$.

Proof. Choose a pair $(\mathbf{A}, \mathbf{B})$ such that condition $\mathrm{H} 0$ is satisfied for $\mathbf{X}(t, s)$, and define the solution operator $H$ for the equivalent augmented system $\left(\mathbf{E}_{\lambda}\right)$. Using Lemma 5 and Lemma 6 , we readily find that $\mu\left(D_{2} H\left(\phi^{*}, \lambda^{*}\right)\right)=-g_{01}$. Similarly, we find

$$
\mu\left(D_{1}^{2} H\left(\phi^{*}, \lambda^{*}\right)\left[V_{\lambda^{*}} \xi_{1}\right]^{2}\right)=\mu\left(D^{(2,0)} H\left(\phi^{*}, \lambda^{*}\right)\left[X_{\lambda^{*}} \xi_{1}\right]^{2}\right)=-g_{20}
$$

The result follows by applying Theorem I.4.1 of [Kielhofer, 2012] or Theorem A.2 of [Pötzsche, 2010]. 
Theorem 6 [Bifurcation with 1-dimensional kernel]. Let $\Lambda \subseteq \mathbb{R}, m \geq 2$. Suppose H1-H5 hold with $p=$ $r=1$ and the transversality condition

$$
g_{11}:=\int_{\mathbb{R}}\left\langle Y_{\lambda^{*}} \xi_{1}^{\prime}, D_{2} D_{3} f\left(t, 0, \lambda^{*}\right) V_{\lambda^{*}} \xi_{1}\right\rangle d t+\sum_{k \in \mathbb{Z}}\left\langle Y_{\lambda^{*}}\left(\tau_{k}^{+}\right) \xi_{i}^{\prime}, D_{1} D_{2} J_{k}\left(0, \lambda^{*}\right) V_{\lambda^{*}}\left(\tau_{k}^{+}\right) \xi_{1}\right\rangle \neq 0,
$$

is satisfied. Then, the trivial solution bifurcates at $\lambda^{*}$. In particular, there exists $\rho>0$ and open convex neighbourhoods $U \subseteq B P C^{1}(\mathbb{R}, \Omega)$ of $0, \lambda_{0} \subseteq \Lambda$ of $\lambda^{*}$, and nontrivial $C^{m}$ functions $\phi:(-\rho, \rho) \rightarrow U$, $\lambda:(-\rho, \rho) \rightarrow \Lambda_{0}$ such that

- $\phi(0)=0, \lambda(0)=\lambda^{*}$, and $\dot{\phi}=X_{\lambda^{*}} \xi_{i}, \dot{\lambda}(0)=0$,

- each $\phi(s)$ is a complete solution of $\left(E_{\lambda(s)}\right)$ in $B P C^{1}(\mathbb{R}, \Omega)$.

Proof. Choose a pair $(\mathbf{A}, \mathbf{B})$ such that condition H0 is satisfied for $\mathbf{X}(t, s)$, and define the solution operator $H$ for the equivalent augmented system $\left(\mathbf{E}_{\lambda}\right)$. We use Lemma 5 and 6 to find $\mu\left(D_{1} D_{2} H\left(0, \lambda^{*}\right)\right)=-g_{11}$, which is nonzero by the transversality assumption. We apply Theorem I.5.1 of [Kielhofer, 2012] or Theorem A.3/A.4 of [Pötzsche, 2010], completing the proof.

The following corollaries are proven in an identical fashion to the above three theorems, using results of Section 1.6 of [Kielhofer, 2012] or Theorems A.3/A.4 of [Pötzsche, 2010].

Corollary 5.1 [Transcritical bifurcation]. Let the conditions of Theorem 6 hold. If additionally

$$
g_{20}:=\int_{\mathbb{R}}\left\langle Y_{\lambda^{*}} \xi_{1}^{\prime}, D_{2}^{2} f\left(t, 0, \lambda^{*}\right)\left[V_{\lambda^{*}} \xi_{1}\right]^{2}\right\rangle d t+\sum_{k \in \mathbb{Z}}\left\langle Y_{\lambda^{*}}\left(\tau_{k}^{+}\right) \xi_{i}{ }^{\prime}{ }^{\prime} D_{1}^{2} J_{k}\left(0, \lambda^{*}\right)\left[V_{\lambda^{*}}\left(\tau_{k}^{+}\right) \xi_{1}\right]^{2}\right\rangle \neq 0,
$$

one has $\dot{\lambda}=-\frac{g_{20}}{2 g_{11}}$, and the following holds locally in $\Lambda_{0}$ : equation $\left(E_{\lambda}\right)$ has a unique nontrivial complete bounded solution $\phi_{\lambda}$ for $\lambda \neq \lambda^{*}$, and 0 is the unique complete bounded solution of $\left(E_{\lambda^{*}}\right)$.

Corollary 5.2 [Pitchfork bifurcation]. Let the conditions of Theorem 6 hold. If additionally $g_{20}=0$ and

$$
g_{30}:=\int_{\mathbb{R}}\left\langle Y_{\lambda^{*}} \xi_{1}^{\prime}, D_{2}^{3} f\left(t, 0, \lambda^{*}\right)\left[V_{\lambda^{*}} \xi_{1}\right]^{3}\right\rangle d t+\sum_{k \in \mathbb{Z}}\left\langle Y_{\lambda^{*}}\left(\tau_{k}^{+}\right) \xi_{i}^{\prime}, D_{1}^{3} J_{k}\left(0, \lambda^{*}\right)\left[V_{\lambda^{*}}\left(\tau_{k}^{+}\right) \xi_{1}\right]^{3}\right\rangle \neq 0,
$$

one has $\dot{\lambda}=0, \ddot{\lambda}=-\frac{g_{30}}{3 g_{11}}$, and the following holds locally in $\Lambda_{0}$ :

- Subcritical case: If $g_{30} / g_{01}>0$, there exists $\Omega_{0} \subseteq \Omega$ open and containing the image of $\phi^{*}$ such that the unique bounded solution of $\left(E_{\lambda}\right)$ in $B P C\left(\Omega_{0}\right)$ for $\lambda \geq \lambda^{*}$ is the trivial solution, while for $\lambda<\lambda^{*}$, there are exactly two nontrivial complete solutions in $B P C\left(\Omega_{0}\right)$.

- Supercritical case: If $g_{30} / g_{01}<0$, there exists $\Omega_{0} \subseteq \Omega$ open and containing the image of $\phi^{*}$ such that the unique bounded solution of $\left(E_{\lambda}\right)$ in $B P C\left(\Omega_{0}\right)$ for $\lambda \leq \lambda^{*}$ is the trivial solution, while for $\lambda<\lambda^{*}$, there are exactly two nontrivial complete solutions in $B P C\left(\Omega_{0}\right)$.

\section{Applications to scalar impulsive systems}

We begin with a general, computable necessary condition for bifurcation in nonlinear scalar impulsive systems that may serve as a first-order test for the presence of a bifurcation. We then provide two examples of this form; the impulsive Bernoulli equation (as introduced in [Akhmet \& Kashkynbayev, 2016]) and a logistic equation with harvesting. Following this, we state a corollary pertaining to sufficient conditions for bifurcation in the sense of [Rasmussen, 2007].

\subsection{Application: A necessary conditon for bifurcation of bounded solutions in nonlinear scalar impulsive differential equations}

Suppose system $\left(E_{\lambda}\right)$ is scalar; $x \in \Omega \subseteq \mathbb{R}, \lambda \in \Lambda$. Let assumptions H1-H2 hold and let $\phi^{*}$ be a given bounded complete solution of $\left(E_{\lambda^{*}}\right)$. Suppose $\phi^{*}$ bifurcates at $\lambda^{*}$. Then, by proposition $3, \phi^{*}$ is nonhyperbolic. By fully characterizing hyperbolicity of the solution in terms of the impulsive differential equation, we can provide an elegant necessary bifurcation condition. Namely, we will prove the following. 
Theorem 7. Let $\left(E_{\lambda}\right)$ be scalar and satisfy hypotheses H1-H2. Introduce the function $\gamma:\{(t, s) \in \mathbb{R} \times \mathbb{R}:$ $t>s\} \rightarrow \mathbb{R}$ defined by

$$
\gamma(t, s)=\frac{1}{t-s}\left[\int_{s}^{t} D_{2} f\left(u, \phi^{*}(u), \lambda^{*}\right) d u+\sum_{\tau_{k} \in[s, t)} \log \left|1+D_{1} J_{k}\left(\phi^{*}\left(\tau_{k}\right), \lambda^{*}\right)\right|\right] .
$$

Let $\lambda^{*} \in \Lambda$. The solution $\phi^{*}$ of $\left(E_{\lambda^{*}}\right)$ is nonhyperbolic if and only if

$$
\liminf _{t-s \rightarrow \infty} \gamma(t, s) \leq 0 \leq \limsup _{t-s \rightarrow \infty} \gamma(t, s) .
$$

In particular, if $\phi^{*}$ bifurcates at $\lambda^{*}$ then equation (27) is satisfied.

Proof. $\quad \phi^{*}$ is nonhyperbolic if and only if the variational equation does not posess exponential dichotomy. The variational equation is precisely

$$
\begin{aligned}
\dot{y} & =D_{2} f\left(t, \phi^{*}(t), \lambda^{*}\right) y, \quad t \neq \tau_{k} \\
\Delta y & =D_{1} J_{k}\left(\phi^{*}\left(\tau_{k}\right), \lambda^{*}\right), \quad t=\tau_{k},
\end{aligned}
$$

which has the fundamental matrix solution $X(t, s)$ that can be written as

$$
X(t, s)=\exp \left[\int_{s}^{t} D_{2} f\left(u, \phi^{*}(u), \lambda^{*}\right) d u+\operatorname{sign}(t-s) \sum_{\tau_{k} \in i(s, t)} \log \left(1+D_{1} J_{k}\left(\phi^{*}\left(\tau_{k}\right), \lambda^{*}\right)\right)\right],
$$

and $i(s, t)=[s, t)$ if $s \leq t$ and $i(s, t)=[t, s)$ if $s \geq t$. Since $X(t, s)$ is scalar, there is an exponential dichotomy if and only if either $|X(t, s)| \leq K e^{-\alpha(t-s)}$ or $|X(s, t)| \leq K e^{-\alpha(s-t)}$ for $t \leq s$ and constants $K \geq 1$ and $\alpha>0$. Also,

$$
|X(t, s)|=\exp \left[\int_{s}^{t} D_{2} f\left(u, \phi^{*}(u), \lambda^{*}\right) d u+\operatorname{sign}(t-s) \sum_{\tau_{k} \in i(s, t)} \log \left|1+D_{1} J_{k}\left(\phi^{*}\left(\tau_{k}\right), \lambda^{*}\right)\right|\right],
$$

Notice that, due to our explicit representation of $|X(t, s)|$, the case of a stable exponential dichotomy, $|X(t, s)| \leq K e^{-\alpha(t-s)}$ for $t \geq s$, is equivalent to having

$$
\gamma(t, s) \leq \frac{\log K}{t-s}-\alpha .
$$

If the above inequality holds for all $t \geq s$ so that that the stable exponential dichotomy occurs, then in particular, one has

$$
\gamma^{+}=\limsup _{t-s \rightarrow \infty} \gamma(t, s)<0 .
$$

For the converse, suppose that the above limit superior is negative. Let $\epsilon>0$ be small enough so that $\gamma^{+}+\epsilon<0$. Then, there exists $N>0$ such that for all $t-s>N$, one has

$$
\gamma(t, s)<\frac{\log K}{t-s}+\left(\gamma^{+}+\epsilon\right)
$$

for any $K \geq 1$. Define the constant $K$ by

$$
\log K=\max \left\{0,\left(-\gamma^{+}+\sup _{t-s \leq N} \gamma(t, s)\right) N\right\}
$$

Then, for $t-s \leq N$, one has

$$
\gamma(t, s) \leq \sup _{t-s \leq N} \gamma(t, s) \leq \frac{\log K}{N}+\gamma^{+} \leq \frac{\log K}{t-s}+\left(\gamma^{+}+\epsilon\right) .
$$

It follows that when $\gamma^{+}<0$, there exists constants $K \geq 1$ and $\alpha=-\left(\gamma^{+}+\epsilon\right)>0$ such that $\gamma(t, s) \leq \frac{\log K}{t-s}-\alpha$ for all $t \geq s$. We conclude that that $X(t, s)$ has a stable exponential dichotomy if and only if $\gamma^{+}<0$. 
For the case of an unstable exponential dichotomy, the inequality $|X(s, t)| \leq K e^{-\alpha(t-s)}$ for $t \geq s$ is equivalent to

$$
\gamma(t, s) \geq-\frac{\log K}{t-s}+\alpha
$$

If the above holds for all $t \geq s$ so that there is an unstable exponential dichotomy, then in particular, one has

$$
\gamma^{-}=\liminf _{t-s \rightarrow \infty} \gamma(t, s)>0
$$

Conversely, if $\gamma^{-}>0$, let $\epsilon>0$ be small enough so that $\gamma^{-}-\epsilon>0$. Then, there exists $N>0$ such that for all $t-s \geq N$, one has

$$
\gamma(t, s)>-\frac{\log K}{t-s}+\left(\gamma^{-}-\epsilon\right)
$$

for all $K \geq 1$. Define the constant $K$ by

$$
\log K=\max \left\{0,\left(\gamma^{-}-\inf _{t-s \leq N} \gamma(t, s)\right) N\right\} .
$$

Then, it follows that for all $t-s \leq N$,

$$
\gamma(t, s) \geq \inf _{t-s \leq N} \gamma(t, s) \geq-\frac{\log K}{N}+\gamma^{-} \geq \frac{-\log K}{t-s}+\left(\gamma^{-}-\epsilon\right)
$$

It follows that when $\gamma^{-}>0$, there exists constants $K \geq 1$ and $\alpha=\gamma^{-}-\epsilon>0$ such that $\gamma(t, s) \geq-\frac{\log K}{t-s}+\alpha$ for all $t \geq s$. We conclude that that $X(t, s)$ has an unstable exponential dichotomy if and only if $\gamma^{-}>0$.

Since $X(t, s)$ has an exponential dichotomy if and only if it has either a stable or unstable exponential dichotomy, we have that $\phi^{*}$ is nonhyperbolic if and only if $X(t, s)$ has neither a stable nor unstable exponential dichotomy. By the above, this is equivalent to having $\gamma^{-} \leq 0 \leq \gamma^{+}$.

\subsubsection{Example: Impulsive Bernoulli equations}

Theorem 7 illustrates that the sufficient conditions for transcritical and pitchfork bifurcation patterns identified in [Akhmet \& Kashkynbayev, 2013, 2016] are also necessary. Indeed, in [Akhmet \& Kashkynbayev, 2016], the authors prove that a sufficient condition for bifurcation of the trivial solution of the impulsive Bernoulli equation

$$
\begin{aligned}
\dot{x} & =p(t) x-q(t) x^{n}, & & \neq \tau_{k} \\
\Delta x & =-x+\frac{x}{\left(c_{k}+d_{k} x^{n-1}\right)^{\frac{1}{n-1}}}, & & t=\tau_{k},
\end{aligned}
$$

with $n \geq 2$ is that the parameter

$$
\gamma=\limsup _{t-s \rightarrow \infty} \frac{1}{t-s}\left[\int_{s}^{t}(1-n) p(u) d u+\sum_{s \leq \tau_{k}<t} \log c_{k}\right]
$$

passes through zero. The above can be equivalently written as

$$
(1-n) \limsup _{t-s \rightarrow \infty} \frac{1}{t-s}\left[\int_{s}^{t} p(u) d u+\sum_{s \leq \tau_{k}<t} \log c_{k}^{-\frac{1}{n-1}}\right] .
$$

Since the variational equation for $(31)$ is precisely

$$
\begin{array}{rlrl}
\dot{y} & =p(t) y, & t \neq \tau_{k} \\
\Delta y & =\left(-1+c_{k}^{\frac{-1}{n-1}}\right) y, & t & t=\tau_{k},
\end{array}
$$


we see that (32) is a rescaled version of $\lim \sup _{t-s \rightarrow \infty} \gamma(t, s)$ as defined in Theorem 7 , where the scaling factor $1-n$ comes from the Bernoulli transformation the authors use to derive their bifurcation condition.

\subsubsection{Example: Nonhyperbolicity in a logistic-type differential equation with impulsive harvesting}

Consider the logistic equation with time-dependent intrinsic growth $g(t)$, carrying capacity $K(t)$ and impulsive harvesting at times $\tau_{k}$ with linear, parameter-dependent harvesting:

$$
\begin{aligned}
\dot{x} & =g(t) x(1-x / K(t)), & & t \neq \tau_{k} \\
\Delta x & =-\lambda x, & & t=\tau_{k},
\end{aligned}
$$

where $\lambda \in(0,1)$ is the harvesting effort. For the trivial solution (extinction state) $\phi^{*}=0$, the function $\gamma(t, s)$ of Theorem 7 can be written

$$
\gamma(t, s)=\frac{1}{t-s}\left[\int_{s}^{t} g(u) d u+\log (1-\lambda) \#\left\{\tau_{k} \in[s, t)\right\}\right]
$$

If the species grows seasonally, we might assume $g \geq 0$ is periodic ${ }^{4}$ with mean growth rate $\mu$. In this case, if we denote

$$
\delta^{-}=\liminf _{t-s \rightarrow \infty} \frac{\#\left\{\tau_{k} \in[s, t)\right\}}{t-s}, \quad \delta^{+}=\limsup _{t-s \rightarrow \infty} \frac{\#\left\{\tau_{k} \in[s, t)\right\}}{t-s},
$$

it follows by Theorem 7 that 0 is nonhyperbolic if and only if

$$
\mu+\log (1-\lambda) \delta^{-} \leq 0 \leq \mu+\log (1-\lambda) \delta^{+} .
$$

However, since $\lambda \in(0,1)$, this can only occur if the above holds with equality. Therefore, if the trivial solution bifurcates at $\lambda$, then we must have

$$
\lambda=1-\exp \left(-\frac{\mu}{\delta^{+}}\right)
$$

\subsection{Application: All-time bifurcation in nonlinear scalar impulsive systems}

Theorem 7 can provide a sufficient condition for bifurcation as well, albeit for a slightly different definition of bifurcation [Rasmussen, 2007].

Definition 6.1. Let $\phi^{*}$ denote a complete $\lambda$-invariant solution of $\left(E_{\lambda}\right)$. Let $x\left(t, t_{0}, x_{0}, \lambda\right)$ denote the solution of $\left(E_{\lambda}\right)$ with initial condition $x\left(t_{0}, t_{0}, x_{0}, \lambda\right)=x_{0}$, and let $d(A, B)=\sup _{a \in A} \inf _{b \in B}|a-b|$ denote the Hausdorff semidistance. We say that $\phi^{*}$

- is all-time attractive if there exists $\eta>0$ such that

$$
\lim _{t \rightarrow \infty} \sup _{t_{0} \in \mathbb{R}} d\left(x\left(t+t_{0}, t_{0}, B_{\eta}\left(\phi^{*}\left(t_{0}\right) \lambda\right), \lambda\right), \phi^{*}\left(t+t_{0}\right)\right)=0,
$$

and in this case, the supremum of all such $\eta$ is denoted $\mathcal{A}_{\phi^{*}}^{\lambda}$, the radius of all-time attraction of $\phi^{*}$.

- is all-time repulsive if there exists $\eta>0$ such that

$$
\lim _{t \rightarrow \infty} \sup _{t_{0} \in \mathbb{R}} d\left(x\left(t_{0}-t, t_{0}, B_{\eta}\left(\phi^{*}\left(t_{0}\right) \lambda\right), \lambda\right), \phi^{*}\left(t_{0}-t\right)\right)=0,
$$

and in this case, the supremum of all such $\eta$ is denoted $\mathcal{R}_{\phi^{*}}^{\lambda}$, the radius of all-time repulsion of $\phi^{*}$.

\footnotetext{
$\overline{{ }^{4} \text { Or, more generally, that } \lim \sup _{t-s \rightarrow \infty} \int_{s}^{t} g(u) d u}=\mu=\liminf _{t-s \rightarrow \infty} \int_{s}^{t} g(u) d u$.
} 
- undergoes an all-time bifurcation at $\lambda^{*}$ if the following holds locally for $\lambda$ near $\lambda^{*}$ : $\phi^{*}$ is all-time attractive for $\lambda<\lambda^{*}$ (resp. $\lambda>\lambda^{*}$ ), all-time repulsive for $\lambda>\lambda^{*}$ (resp. $\lambda<\lambda^{*}$ ), and the radii of all-time attraction and repulsion satisfy

$$
\lim _{\lambda \nearrow \lambda^{*}} \mathcal{A}_{\phi^{*}}^{\lambda}=0, \quad \lim _{\lambda \searrow \lambda^{*}} \mathcal{R}_{\phi^{*}}^{\lambda}=0
$$

(resp. with sidedness of limits reversed).

The following corollary provides a sufficient condition for a loss (or gain) of stability due to parameter variation, based Theorem 7 .

Corollary 6.1. Let $\phi^{*}$ denote a $\lambda$-invariant solution of the scalar equation $\left(E_{\lambda}\right)$. Let hypotheses H1-H2 hold, and let $\gamma(t, s ; \lambda)$ denote the function (26) associated to the solution $\phi^{*}$, where we now make explicit the dependence on the parameter $\gamma$. Suppose for some $\lambda^{*} \in \mathbb{R}$, the following holds locally near $\lambda^{*}$ :

$$
\begin{array}{ll}
\limsup _{t-s \rightarrow 0} \gamma(t, s, \lambda)<0, & \lambda<\lambda^{*} \\
\liminf _{t-s \rightarrow 0} \gamma(t, s, \lambda)>0, & \lambda>\lambda^{*},
\end{array}
$$

(resp. inequalities involving $\lambda$ and $\lambda^{*}$ reversed). Then $\phi^{*}$ is all-time attractive for $\lambda<\lambda^{*}$, all-time repulsive for $\lambda>\lambda^{*}$ (resp. inequalities reversed).

Proof. To obtain the attractivity and repulsivity, apply Theorem 1 and Theorem 2 of [Akhmet \& Kashkynbayev, 2013], taking into account the exponential dichotomies guaranteed for $\lambda<\lambda^{*}$ and $\lambda>\lambda^{*}$ by Theorem 7 and the associated exponential stability of the solution $\phi^{*}$ in the nonlinear system (forward or time-reversed) as in [Bainov \& Simeonov, 1988]

The above conditions do not guarantee an all-time bifurcation occurs. The associated trivialization of radii of all-time attraction and repulsion as in Definition 6.1 require somewhat stronger conditions than those given in the above corollary. Specifically, one may need to impose particular boundedness properties on the second and/or third-order terms of the taylor expansion of the vector field and difference equation; see [Akhmet \& Kashkynbayev, 2016] for relevant conditions.

\section{Examples of bifurcations of bounded solutions in planar systems}

In this section, we provide two examples of transcritical and pitchfork bifurcation as presented in Section 5.2. These two examples are planar, minimal, and somewhat contrived, serving only to provide explicitly computable examples for the application of Corollaries 5.1-5.2.

\subsection{Example: Transcritical Bifurcation}

Consider the impulsive system

$$
\begin{aligned}
\dot{x} & =\alpha \tanh (t) x+\gamma y^{2}, & & t \neq \tau_{k} \\
\dot{y} & =\beta \tanh (t) y, & & t \neq \tau_{k} \\
\Delta x & =a \lambda x+b \lambda y, & & t=\tau_{k} \\
\Delta y & =c \lambda x, & & t=\tau_{k},
\end{aligned}
$$

with constants $\alpha, \beta, a, b, c \in \mathbb{R}, b, \gamma \neq 0, \alpha>0>\beta$. We see that $(x, y)=(0,0)$ is a a solution. The variational equation about the trivial solution for $\lambda=0$ has an exponential dichotomy on half-lines with projectors

$$
P=\left[\begin{array}{ll}
0 & 0 \\
0 & 1
\end{array}\right], \quad Q=\left[\begin{array}{ll}
1 & 0 \\
0 & 0
\end{array}\right]
$$


on $[0, \infty)$ and $(-\infty, 0]$ respectively. Therefore, $\mathcal{R} P=\mathcal{N} Q=\mathbb{R}\left[\begin{array}{ll}0 & 1\end{array}\right]^{T}$. By Theorem 2 , the index is zero and the kernel of $\mathcal{L}$ is one-dimensional. We choose $\xi_{1}=\left[\begin{array}{ll}0 & 1\end{array}\right]^{T}$ and $\xi_{1}^{\prime}=\left[\begin{array}{ll}1 & 0\end{array}\right]^{T}$, and we have

$$
V_{0}(t)=\left[\begin{array}{cc}
\exp (\alpha \log \cosh (t)) & 0 \\
0 & \exp (\beta \log \cosh (t)))
\end{array}\right] .
$$

Denote $f(t,(x, y), \lambda)=\left(\alpha \tanh (t) x+y^{2}, \beta \tanh (t) y\right)$ and $J_{k}((x, y), \lambda)=(a \lambda x+b \lambda y, c \lambda x)$. Since $D_{2} D_{3} f=0$, we compute $g_{11}$ as

$$
\begin{aligned}
g_{11} & =\sum_{k \in \mathbb{Z}}\left\langle V_{0}^{-1 *} \xi_{1}^{\prime}, D_{1} D_{2} J_{k}(0,0) V_{0}\left(\tau_{k}^{+}\right) \xi_{i}\right\rangle \\
& =\sum_{k \in \mathbb{Z}}\left[\begin{array}{ll}
1 & 0
\end{array}\right] e^{-\alpha \log \cosh \left(\tau_{k}\right)}\left[\begin{array}{ll}
a & b \\
c & 0
\end{array}\right]\left[\begin{array}{l}
0 \\
1
\end{array}\right] e^{\beta \log \cosh \left(\tau_{k}\right)} \\
& =b \sum_{k \in \mathbb{Z}} \exp \left((-\alpha+\beta) \log \cosh \left(\tau_{k}\right)\right) \\
& \neq 0
\end{aligned}
$$

Therefore, a bifurcation occurs at $\lambda=0$ by Theorem 6 . Since $D_{1}^{2} J_{k}=0$, we compute the coefficient $g_{20}$ as

$$
\begin{aligned}
& g_{20}=\int_{\mathbb{R}}\left\langle V_{0}^{-1 *} \xi_{1}^{\prime}, D_{2}^{2} f(t, 0,0)\left[V_{0}(t) \xi_{1}\right]^{2}\right\rangle \\
& =\int_{\mathbb{R}}\left[\begin{array}{ll}
1 & 0
\end{array}\right] e^{-\alpha \log \cosh (t)}\left[\begin{array}{cc}
0 & 2 \gamma \\
0 & 0
\end{array}\right]\left[\begin{array}{l}
0 \\
1
\end{array}\right] e^{\beta \log \cosh (t)} d t \\
& =2 \gamma \int_{\mathbb{R}} \exp ((-\alpha+\beta) \log \cosh (t)) d t \\
& \neq 0 \text {. }
\end{aligned}
$$

By Corollary 5.1, the trivial undergoes a transcritical bifurcation at $\lambda=0$.

\subsection{Example: Pitchfork Bifurcation}

We consider a similar impulsive system to the previous example:

$$
\begin{aligned}
\dot{x} & =\alpha \tanh (t) x+\gamma y^{3}, & & t \neq \tau_{k} \\
\dot{y} & =\beta \tanh (t) y, & & t \neq \tau_{k} \\
\Delta x & =a \lambda x+b \lambda y, & & t=\tau_{k} \\
\Delta y & =c \lambda x, & & t=\tau_{k},
\end{aligned}
$$

with constants $\alpha, \beta, a, b, c \in \mathbb{R}, b, \gamma \neq 0, \alpha>0>\beta$. As before, the index of the associated linearized solution operator is zero and the kernel is one-dimensional. The coefficient $g_{11}$ is the same as in the previous example. Overall, we find

$$
g_{11}=b \sum_{k \in \mathbb{Z}} \exp \left((-\alpha+\beta) \log \cosh \left(\tau_{k}\right)\right), \quad g_{30}=6 \gamma \int_{\mathbb{R}} \exp ((-\alpha+\beta) \log \cosh (t)) d t,
$$

both of which are nonzero. Therefore, by Corollary 5.2, the trivial solution undergoes a pitchfork bifurcation at $\lambda=0$. The sign of $\gamma / b$ determines whether the bifurcation is subcritical (positive) or supercritical (negative).

\section{Conclusion}

In this article, we have provided a necessary condition for the bifurcation of a bounded solution in nonlinear impulsive differential equations (Proposition 3) and derived sufficient conditions for classical fold, transcritical and pitchfork bifurcations (Section 5), among others. The former result allowed us to derive 
an analytically precise necessary condition for bifurcation in nonlinear scalar impulsive systems (Theorem 7), and it was shown that this condition is consistent with sufficient conditions for bifurcations that have been derived by other authors (Section 6.1.1). We also provided a result relating to all-time bifurcation of scalar impulsive systems (Corollary 6.1). We concluded with two two-dimensional examples that illustrate our sufficient conditions for transcritical and pitchfork bifurcations.

The approach to all of the above results is ultimately based on abstract bifurcation theory, specifically, Lyapunov-Schmidt reduction. The solution operator $H$ defined in equation (6) differs from the operator defined in [Pötzsche, 2010] for ordinary differential equations in that it is explicitly an integral operator as opposed to a differential operator. This distinction is required because it is not possible to incorporate discontinuities in state (the impulses) into a differential operator and maintain the desirable Banach space setting that makes the Lyapunov-Schmidt reduction possible. The caveat is that we must introduce arbitrary linear factors (Equation $\left(\mathbf{E}_{\lambda}\right)$ ) for the operator $H$ to be well-defined. However, as expected, the linear factors have no impact on the necessary or sufficient conditions for bifurcation.

\section{References}

Akhmet, M. [2010] Principles of discontinuous dynamical systems, (Springer-Verlag, New York)

Akhmet, M.U., Kashkynbayev, A. [2013] Non-autonomous bifurcation in impulsive systems. Electr. J. Qual. Theory Differ. Equ. 74

Akhmet, M.U., Kashkynbayev, A. [2013] Nonautonomous transcritical and pitchfork bifurcations in impulsive systems. Miskolc Math. Notes 14, 737-748

Akhmet, M. and Kashkynbayev, A. [2016] Nonautonomous Bifurcations in Nonlinear Impulsive Systems, Differ Equ Dyn Syst. doi:10.1007/s12591-016-0309-7

Simeonov, P.S and Bainov, D.D. [1988] Exponential stability of the solutions of the initial-value problem for systems with impulse effect Journal of Computational and Applied Mathematics 23, 353-365

Bainov, D.D. and Simeonov, P.S. [1993] Impulsive Differential Equations: Periodic Solutions and Applications. (Longman Scientific \& Technical, Burnt Mill)

Chow, S.-N. and Hale, J.K. [1996] Methods of Bifurcation Theory. (Grundlehren der mathematischen Wissenschaften 251, Springer, Berlin etc.)

Church, K.E.M. and Smith?, R.J. [2016] Comparing malaria surveillance with periodic spraying in the presence of insecticide-resistant mosquitoes: Should we spray regularly or based on human infections? Mathematical Biosciences 276, 145-163.

Galbusera, L. and Pasquali, S. [2015] Analysis and constrained optimal impulsive control of a Holling-II type trophic system with two sources, Journal of the Franklin Institute 352, 2728-2749

Georgescu, P., Zhang, H. and Chen, L. [2008] Bifurcation of nontrivial periodic solutions for an impulsively controlled pest management model, Applied Mathematics and Computation 202, 675-687.

Kielhöfer, H. [2012] Bifurcation Theory: An Introduction with Applications to Partial Differential Equations, Second Edition, Applied Mathematical Sciences 156. (Springer)

Kiskinov, H. et. al [2015] Weighted exponential Dichotomy of the Solutions of linear impulsive differential Equations in a Banach Space, Rostock. Math. Kolloq. 69, 3-17

Lang, S. [1993] Real and functional analysis, Graduate Texts in Mathematics 142. (Springer, Berlin)

Langa, J.A., Robinson, J.C., Suarez, A. [2002] Stability, Instability and bifurcation phenomena in nonautonomous differential equations. Nonlinearity 15, 1-17.

Langa, J.A., Robinson, J.C., Suarez, A. [2006] Bifurcation in non-autonomous scalar equations. J. Differ. Equ. 221, 1-35.

Li, A., Feng, E. and Wang, L. [2009] Impulsive optimal control model for the trajectory of horizontal wells, Journal of Computational and Applied Mathematics 223, 893-900

Liu, Y. [2015] Bifurcation techniques for a class of boundary value problems of fractional impulsive differential equations. J. Nonlinear Sci. Appl. 8, 340-353.

Niu, Y. and Yan, B. [2016] Global structure of solutions to boundary-balue problems of impulsive differential equations, Electronic Journal of Differential Equations, Vol. 2016, No. 55, 123.

Liu, Y. and ORegan, D. [2011] Multiplicity results using bifurcation techniques for a class of boundary value 
problems of impulsive differential equations, Commu Nonlinear Sci Nummer Simulat 16, 1769-1775.

Palmer, K. J. [1984] Exponential dichotomies and transversal homoclinic points, J. Diff. Eqns. 55, 225-256.

Pötzsche, C. [2010] Nonautonomous bifurcation of bounded solutions I: a Lyapunov-Schmidt approach, Discrete and Continuous Dynamical Systems - Series B 14(2), 739-776.

Rasmussen, M. [2007] Attractivity and Bifurcation for nonautonomous dynamical systems. (Springer-Verlag, Berlin)

Ruan, S. and Zhang, W. [2005] Exponential Dichotomies, the Fredholm Alternative, and Transverse Homoclinic Orbits in Partial Functional Differential Equations, Journal of Dynamics and Differential Equations 17(4), 759-777.

Zeidler, E. [1993] Nonlinear functional analysis and its applications I (Fixed-point theorems). (Springer, Berline etc.)

Zhao, Z. and Chen, L. [2009] Chemical chaos in enzyme kinetics, Nonlinear Dyn 57, 135-142

Zhao, Z., Yang, L. and Chen, L. [2009] Bifurcation of nontrivial periodic solutions for a biochemical model with impulsive perturbations, Applied Mathematics and Computation 215, 2806-2814. 